\title{
Calibrating image roughness by estimating Lipschitz exponents, with applications to image restoration
}

\author{
Alfred S. Carasso \\ Mathematical and Computational Sciences Division \\ András E. Vladár \\ Precision Engineering Division \\ National Institute of Standards and Technology, \\ Gaithersburg, MD 20899 \\ Phone 301-975-2705. FAX 301-975-3553. \\ Email_alfred.carasso@nist.gov, andras.vladar@nist.gov
}




\begin{abstract}
Most images $f(x, y)$ are not smoothly differentiable functions of $x$ and $y$, but display edges, localized singularities, and other significant fine scale roughness or texture. Correct characterization and calibration of image roughness is vital in many image processing tasks. The $L^{1}$ Lipschitz exponent $\alpha$, where $0<\alpha \leq 1$, measures fine scale image roughness provided the image is relatively noise free. This paper describes a recently developed mathematical technique for estimating $\alpha$. The method is based on successively blurring the image by convolution with increasingly narrower Gaussians, using commonly available Fast Fourier Transform algorithms.

Instructive examples are used to illustrate the quantitative changes in $\alpha$ that occur when an image is either degraded or restored. Of particular interest are the documented changes in $\alpha$ that accompany APEX blind deconvolution of real images from the Hubble space telescope, from MRI and PET brain scans, and from state of the art nanoscale Scanning Electron Microscopy. (APEX is the actual name of a blind deconvolution procedure, and is not an acronym or abbreviation).

Additional applications include monitoring of image sharpness and imaging performance in imaging systems, evaluation of image reconstruction software quality, detection of abnormal fine structure in biomedical images, and monitoring of surface finish in industrial applications.
\end{abstract}

Subject terms image roughness; image metrology; Lipschitz exponents; image restoration; APEX deconvolution; Hubble telescope; MRI and PET brain scans; nanoscale microscopy. 


\section{Introduction}

This paper discusses an effective mathematical framework for quantifying the unsmoothness of images, and then applies this methodology to some significant questions in image restoration.* An elegant computational technique, based on Fast Fourier Transform (FFT) algorithms, translates this theory into an important and practical image metrology tool. Interesting real images from such fields as astronomy, electron microscopy, and brain research provide valuable illustrative examples. In all figures, the axis label ' $\log u$ ' refers to the natural logarithm of $u$.

Most natural images $f(x, y)$ display edges, localized sharp features, and other significant fine scale details or texture, and cannot be modeled as smoothly differentiable functions of $x$ and $y$. In many digital image processing tasks, it is necessary to provide prior information that specifies the degree of unsmoothness in the unknown desired true image. If an image is incorrectly postulated to be too smooth, the processing algorithm may produce a smoothed out version of the true image, in which critical diagnostic information has been lost. The class $B V\left(R^{2}\right)$ of functions of bounded variation includes functions with sharp edges, as well as smooth continuously differentiable functions. That class has been used extensively to model images over the last fifteen years or so. ${ }^{1-4}$ However, it has become increasingly evident that the class $B V\left(R^{2}\right)$ does not allow for the type of fine scale sharp structures commonly found in natural images, in addition to edges. For this reason, image deblurring based on the $B V\left(R^{2}\right)$ assumption notoriously produces imagery in which important fine scale texture has been severely eroded. This is known as the staircase effect. ${ }^{3-7}$ It is now recognized that significantly wider function classes, such as the so-called Lipschitz classes $\Lambda(\alpha, p, \infty)$, are necessary to adequately describe natural images.

The $L^{1}$ Lipschitz exponent $\alpha$, where $0<\alpha \leq 1$, is a mathematical index that can capture the fine-scale content and degree of unsmoothness in an image, provided that image is relatively noise free. Images that are of bounded variation (including smoothly differentiable images) have $\alpha=1$. The value of $\alpha$ decreases systematically with increasing roughness. Images with significant non differentiable small scale structures typically have $\alpha \ll 1$. One may

*The mathematical and computational techniques described in this paper are covered by U.S. patent application number 10/928,308. Licensing inquiries should be directed to Terry Lynch, jtlynch@nist.gov 
also consider $L^{p}$ spaces with $p>1$.

In a recent paper, ${ }^{7}$ a new computational approach for estimating image Lipschitz exponents was developed, based on blurring the image with successively narrower Lorentzian (Cauchy) probability density functions. This theory was then used in Ref. 7 to construct a new image deblurring method, the so-called Poisson Singular Integral (PSI) method. That method is based on prior knowledge of the $L^{2}$ Lipschitz exponent in the unknown true image. An educated guess for that value, based on knowledge of exponent values for similar images, is often sufficient to obtain useful sharpening. The major result in Ref. 7 is the demonstration that the PSI method can recover texture in cases where the $B V\left(R^{2}\right)$ approach fails completely.

The present paper is based on the use of technically more advantageous Gaussian densities rather than Lorentzian densities. It complements the results in Ref. 7 by exploring further applications of Lipschitz exponents, in particular the quantitative changes in $\alpha$ that occur when an image is either degraded or restored. Of significant interest are the documented changes in $\alpha$ that accompany APEX blind deconvolution of real images. ${ }^{6}$ Examples include images from the Hubble space telescope, from magnetic resonance imaging (MRI) brain scans, from functional cerebral positron emission tomography (PET) imaging, and from state of the art nanoscale scanning electron microscopy (SEM). We also consider synthetically degraded images and compare the changes in Lipschitz exponent that result from competing restoration algorithms.

The above examples indirectly bear upon several other contexts where a simple practical method of quantifying image roughness can be useful. Many imaging systems suffer performance degradation over time and require periodic maintenance. In scanning electron microscopes, the shape of the electron beam changes with time, often without the user's knowledge. Periodic performance testing can be accomplished by monitoring sharpness degradation in the micrograph of a specially designed test object. ${ }^{8}$ Such degradation can be quantified by measuring the increase in Lipschitz exponent. In some imaging systems, a decrease in $\alpha$ might indicate an increase in system noise. Measuring the $\alpha$ of a test image can also be used to evaluate and compare the performance of competing image reconstruction packages. Automatic measurement of $\alpha$ might be useful in monitoring the smoothness of surface finishes in certain industrial applications. In some medical applications, measurement of $\alpha$, along with other appropriate processing, might be helpful in 
prescreening for certain types of abnormalities.

Sections 2-4 develop the mathematical preliminaries, culminating in Eq. (15) which can be evaluated using FFT algorithms. Sections 5-7 illustrate the use of that technique in estimating Lipschitz exponents. Section 8 studies the behavior of Lipschitz exponents as images are synthetically degraded and restored. Section 9 demonstrates the value of that tool in confirming APEX blind sharpening of real blurred imagery. Finally, Section 10 contains the conclusion.

\section{Lipschitz classes and image roughness}

A function $f(x, y) \in B V\left(R^{2}\right)$ may not be differentiable at a given set of points, but it is constrained to satisfy

$$
\int_{\mathbf{R}^{2}}\left|f\left(x+h_{1}, y+h_{2}\right)-f(x, y)\right| d x d y \leq \text { Const }|h|, \quad|h| \rightarrow 0,
$$

where

$$
|h|=\left(h_{1}^{2}+h_{2}^{2}\right)^{1 / 2} .
$$

However, from the standpoint of modeling texture, it is advantageous to consider functions $f(x, y)$ satisfying a weaker constraint, such as

$$
\int_{\mathbf{R}^{2}}\left|f\left(x+h_{1}, y+h_{2}\right)-f(x, y)\right| d x d y \leq \text { Const }|h|^{\alpha}, \quad|h| \rightarrow 0,
$$

where $\alpha$ is fixed, and $0<\alpha<1$. Such an $f$ is said to be of Lipschitz class $\Lambda(\alpha, 1, \infty)$. A function $f(x, y)$ satisfying Eq. (1) necessarily satisfies Eq. (3), but not vice versa in general. Thus $B V\left(R^{2}\right) \subset \Lambda(\alpha, 1, \infty)$.

One may also consider Lipschitz classes $\Lambda(\alpha, p, \infty), \quad 1 \leq p<\infty$, consisting of functions $f(x, y)$ satisfying

$$
\left\{\int_{\mathbf{R}^{2}}\left|f\left(x+h_{1}, y+h_{2}\right)-f(x, y)\right|^{p} d x d y\right\}^{1 / p} \leq \text { Const }|h|^{\alpha}, \quad|h| \rightarrow 0 .
$$

The case $p=2$ is important and was used in Ref. 7 .

An effective method of estimating Lipschitz exponents can be based on blurring the image by successive convolutions with increasingly narrower Gaussians and evaluating the discrete $L^{p}$ norm of the difference between 
the blurred and original images. This $L^{p}$ norm tends to zero as the Gaussian approaches the Dirac $\delta$-function. An important mathematical theorem relates the $L^{p}$ Lipschitz exponent of the image to the rate at which the above $L^{p}$ norm tends to zero. Moreover, that theorem remains valid for the periodized image problem, in which case the convolution can be accomplished quite easily using commonly available FFT algorithms.

\section{The spaces $\Lambda(\alpha, p, \infty)$ and the Gaussian ker- nel}

Define the Fourier transform $\hat{h}(\xi, \eta)$ of $h(x, y) \in L^{1}\left(R^{2}\right)$ by

$$
\mathcal{F}\{h\}=\hat{h}(\xi, \eta) \equiv \int_{R^{2}} h(x, y) \exp \{-2 \pi i(\xi x+\eta y)\} d x d y
$$

For each fixed $t>0$, consider the Gaussian kernel in $R^{2}$

$$
\gamma(x, y, t)=\frac{\exp \left\{-\left(x^{2}+y^{2}\right) / 4 t\right\}}{4 \pi t}, \quad(x, y) \in R^{2} .
$$

We have

$$
\hat{\gamma}(\xi, \eta, t)=\exp \left\{-t\left(\xi^{2}+\eta^{2}\right)\right\} .
$$

For each $t>0$, define the linear operator $G^{t}$ on $L^{p}\left(R^{2}\right), 1 \leq p<\infty$, by

$$
G^{t} f=\int_{R^{2}} \gamma(u, v, t) f(x-u, y-v) d u d v
$$

Since $\gamma(x, y, t)$ approaches the Dirac $\delta$-function as $t$ tends to zero, it is not surprising that $\left\|G^{t} f-f\right\|_{p} \rightarrow 0$ as $t$ tends to zero. However, the rate at which this happens depends on the smoothness (or lack thereof) of $f(x, y)$, and this rate can be used to characterize $f(x, y)$. There is a large literature on that subject. ${ }^{9,10}$ We have from Theorem 4 in Ref. 9,

Theorem 1 Let $G^{t}, t>0$, be the Gauss integral operator in (8), and let $0<\alpha<1,1 \leq p<\infty$. Then, $f \in \Lambda(\alpha, p, \infty)$ if and only if there is a positive constant $K_{p}$ such that

$$
\left\|G^{t} f-f\right\|_{p} \leq K_{p} t^{\alpha / 2}, \quad 0<t \leq 1
$$


The above result can be used to fashion an image analysis tool. Theoretically, given any image $f(x, y)$ in $L^{1}\left(R^{2}\right)$, one could use the Fourier transform in Eq. (5) to form

$$
\mathcal{F}\left\{G^{t} f\right\}=\exp \left\{-t\left(\xi^{2}+\eta^{2}\right)\right\} \hat{f}(\xi, \eta),
$$

for sequences of positive $t$-values tending to zero. Inverse transformation is always possible on account of the factor $\exp \left\{-t\left(\xi^{2}+\eta^{2}\right)\right\}$, and this can be used to produce an infinite sequence of positive numbers

$$
\mu_{n}=\left\{\left\|G^{t_{n}} f-f\right\|_{1} /\|f\|_{1}\right\}, \quad t_{n} \downarrow 0 .
$$

If every such sequence $\left(t_{n}, \mu_{n}\right)$, ultimately lies below the curve $\mu(t)=$ $C_{1} t^{\alpha / 2}$, with $0<t \leq \bar{t}$, and suitably chosen constants $C_{1}>0$ and $0<\alpha<1$, then

$$
\left\|G^{t} f-f\right\|_{1} \leq C_{1}\|f\|_{1} t^{\alpha / 2}, \quad t \downarrow 0,
$$

and $f(x, y) \in \Lambda(\alpha, 1, \infty)$ by Theorem 1 . However, this requires handling infinite domain Fourier integrals and does not lead to a practical procedure.

\section{Periodized problems and FFT algorithms}

A practical procedure can be realized by using the fact that Theorem 1 remains valid in the periodic case. ${ }^{9}$ We now consider the periodized image problem, ${ }^{10-15}$ and obtain the Fourier series analog of Eq. (10). Let $\Omega$ denote the unit square $-1 / 2<x, y \leq 1 / 2$ in $R^{2}$. The image $f(x, y)$ is now viewed as originally defined on $\Omega$ from which it is extended by periodicity to all of $R^{2}$. We redefine the image Fourier transform $\hat{f}(\xi, \eta)$ by

$$
\hat{f}(\xi, \eta)=\int_{\Omega} f(x, y) \exp \{-2 \pi i(\xi x+\eta y)\} d x d y
$$

where $\xi, \eta$ are now integers running from $-\infty$ to $+\infty$. The Poisson Summation Formula, ${ }^{10,16}$ can now be used to construct the periodized Gauss operator $G_{*}^{t}$. That operator is defined by specifying its action on any given image $f(x, y)$. As shown in Ref. $7, G_{*}^{t} f$ is given by the complex Fourier series

$$
G_{*}^{t} f=\sum_{\xi, \eta=-\infty}^{\infty} \exp \left\{-t\left(\xi^{2}+\eta^{2}\right)\right\} \hat{f}(\xi, \eta) \exp \{2 \pi i(x \xi+y \eta)\}
$$


where $\xi, \eta$ are integers running from $-\infty$ to $+\infty$. The factor $\exp \left\{-t\left(\xi^{2}+\eta^{2}\right)\right\}$ assures uniform convergence of the Fourier series in Eq. (14).

Theorem 1 remains valid with $G_{*}^{t}$ replacing $G^{t}$, and using $G_{*}^{t} f$ in Eq. (14) in lieu of $G^{t} f$ in Eq. (8). Next, consider the partial sum

$$
S_{N}(x, y)=\sum_{\xi, \eta=-N}^{N} \exp \left\{-t\left(\xi^{2}+\eta^{2}\right)\right\} \hat{f}(\xi, \eta) \exp \{2 \pi i(x \xi+y \eta)\} .
$$

Because of uniform convergence, $\left\|G_{*}^{t} f-S_{N}\right\|_{p}$ can be made arbitrarily small by choosing $N$ large enough in Eq. (15), and $G_{*}^{t} f$ in Eq. (14) can be approximated by the finite sum $S_{N}$. Given the $2 J \times 2 J$ digitized image $f(x, y)$, the discrete Fourier transform ${ }^{17}$ is now the appropriate numerical tool for approximating the finite Fourier series $S_{J}$. One can use FFT algorithms to approximate the Fourier coefficients $\hat{f}(\xi, \eta),-J \leq \xi, \eta \leq J$, and then apply the filter $\exp \left\{-t\left(\xi^{2}+\eta^{2}\right)\right\}$. An inverse FFT then yields an accurate approximation to $G_{*}^{t} f$ at each of the $2 J \times 2 J$ pixels, for each small $t>0$. We may then examine the discrete $L^{p}$ relative error in Gaussian approximation as $t \downarrow 0$, and locate constants $C_{p}$ and $\alpha$ such that

$$
\left\|G_{*}^{t} f-f\right\|_{p} \leq C_{p}\|f\|_{p} t^{\alpha / 2}, \quad 0<t \leq \bar{t} .
$$

In summary, the results of this section lead to an accurate numerical procedure, based on correct mathematical analysis, for assessing membership in any $\Lambda(\alpha, p, \infty)$ space.

Remark 1: Pitfall at very small $t>0$. We deal with discretely defined high resolution 8-bit images $f(x, y)$, typically of size $512 \times 512$ or $1024 \times 1024$ pixels. Such an $f(x, y)$ may be viewed as a piecewise constant or trigonometric polynomial approximation to the original continuously defined image intensity field $f^{\infty}(x, y)$, or as some other kind of finite dimensional representation of the hypothetical infinite dimensional object $f^{\infty}$. The Lipschitz exponent is predicated on a continuously defined image, and is, in fact, a property of $f^{\infty}(x, y)$. On the other hand, all norms are equivalent on a finite dimensional space. Hence, even if $f^{\infty}(x, y)$ is highly non smooth and not of bounded variation, the discrete total variation norm for $f(x, y)$ is always finite, though it may be very large. To estimate the non smoothness properties of $f^{\infty}(x, y)$ by examination of the finite dimensional representation $f(x, y)$ will require some sagacity. As explained theoretically in Ref. 7 , there is a 
finite dimensionality pitfall in the above Gauss singular integral methodology that requires the exclusion of very small values of $t>0$. As will be amply demonstrated in the examples below, the behavior of $\left\|G_{*}^{t} f-f\right\|_{p}$ at very small $t$ is a spurious artifact that must be ignored. This behavior is disconnected from the true smoothness properties in the image intensity field $f^{\infty}(x, y)$, and it gives a false reading for the Lipschitz exponent $\alpha$.

\section{Application to real images; the character- istic elbow}

Our first example, in Figure 1, is the $512 \times 512$ Giza Pyramids image. The FFT procedure discussed in Section 4 was used to obtain the $L^{1}$ relative errors in Gauss approximation

$$
\mu(t)=\left\|G_{*}^{t} f-f\right\|_{1} /\|f\|_{1}
$$

at 400 values of $t$ given by $t_{n}=0.5(0.95)^{n}, n=1,400$, where $G_{*}^{t} f$ is given by Eq.(14). A plot of $\mu(t)$ versus $t$ on a log-log scale produced the solid curve $A$ in Figure 1. Least squares fitting was used to find the two distinct majorizing dashed straight lines $\Gamma$ and $\Sigma$. For each dashed line, the $y$-axis intercept value obtained by least squares was slightly increased so as to make each line lie visibly above the solid curve $A$; however, the slope of each line remains the same as that obtained from least squares. The curve $A$ exhibits a characteristic elbow shape. It consists of a straight line segment with slope $\approx 1$ beginning near $\log t=-20$ and continuing to near $\log t=-10$. There is then a transition to a different regime, one that is more slowly increasing and that continues to near $\log t=0$. The line $\Gamma$, defined by $\log \mu(t)=7.72+0.988 \log t$, accurately captures the straight line trend in Eq. (17) for very small values of $t$, while being grossly inaccurate at larger values of $t$. It was obtained by excluding data corresponding to $\log t>-12$ from the least squares fit. The line $\Gamma$ implies that $\left\|G_{*}^{t} f-f\right\|_{1}<2253\|f\|_{1} t^{0.988}$ for all $t>0$. As stressed in Remark 1, this correct statement primarily reflects the fact that the $512 \times 512$ Giza Pyramids image lies in a finite dimensional space, ${ }^{7}$ but does not reflect the smoothness properties of the intensity field $f^{\infty}(x, y)$ that gave rise to the digitized Pyramids image. The slope of $\Gamma$ gives a false reading for $\alpha$. Such $\Gamma$ line behavior is a feature of every example in this paper. The 
majorizing dashed straight line $\Sigma$, defined by $\log \mu(t)=-1.31+0.159 \log t$, accurately reflects behavior of Eq. (17) for $-10 \leq \log t \leq-1$, while being grossly inaccurate at very small values of $t$. The line $\Sigma$ was obtained by excluding all data corresponding to $\log t<-10$ from the least squares fit. Note that this still leaves over 160 data points remaining. The behavior along $\Sigma$ indicates that $\left\|G_{*}^{t} f-f\right\|_{1} \leq 0.27\|f\|_{1} t^{0.159}, 0<t \leq 0.1$, and this is taken to be the true behavior in the Pyramids image. From Eq. (9), this implies $\alpha=2.0 \times 0.159=0.318$. Thus the Pyramids image lies in the space $\Lambda(0.318,1, \infty)$, and is not of bounded variation, since this requires $\alpha=1$. Estimates of $\alpha$ in any other discrete $L^{p}$ norm can be obtained similarly. It is recommended that data for very small values of $t$ always be included in log-log plots of $\mu(t)$, so as to enable clear identification of the spurious linear trend, prior to rejecting that part of the data. This procedure of identifying the $\Sigma$ line by using least squares fitting on the 'slowly varying' interval near $\log t=0$, will be used throughout this paper. Blurring with Gaussians leads to better defined elbows than does blurring with Lorentzians.

Remark 2: Parallel computation. The above blurring process with successively narrower Gaussians can obviously be implemented in parallel. For large images, such parallel computation would be significantly more efficient.

\section{$6 \quad$ Lipschitz exponents of some typical images}

An interesting collection of $512 \times 512$ 8-bit images is shown in Figure 2 . These images include natural as well as man-made objects, extending from the nanoscale to the planetary scale. As in Figure 1, least squares fitting was used in the slowly increasing region of the graph of $\log \mu(t)$ vs $\log t$, to find the majorizing $\Sigma$ line in each case. This leads to positive constants $C_{1}$ and $\alpha$ for each image such that

$$
\left\|G_{*}^{t} f-f\right\|_{1} \leq C_{1}\|f\|_{1} t^{\alpha / 2}, \quad 0<t \leq 0.1,
$$

where $G_{*}^{t}$ is the periodized Gauss operator in Eq.(14). As seen in Table 1, these images have $L^{1}$ Lipschitz exponents in the range $0.18<\alpha<0.54$. 


\section{Low Lipschitz exponents}

Clusters of galaxies are examples of objects whose images can have very low Lipschitz exponents. In Figure 3, two $512 \times 512$ images of the Abell 426 (Perseus) cluster are shown side by side. These images were obtained from the NASA Space Telescope Science Institute Digitized Sky Survey. Each image is centered on the exact same point in the sky. However, image (a) covers a 1/2 Mpc square of the sky, while image (b) covers a $1 \mathrm{Mpc}$ square and thus has a higher density of bright pixels. The solid curves A and B are the corresponding plots of $\mu(t)=\left\|G_{*}^{t} f-f\right\|_{1} /\|f\|_{1}$ versus $t$, on a $\log -\log$ scale. Using least squares fitting on $-6 \leq \log t \leq 0$, we find that image (a) has an $L^{1}$ Lipschitz exponent $\alpha=0.116$, while image (b) has $\alpha=0.042$. The corresponding $\Sigma$ lines were not plotted in Figure 3 to avoid clutter. Notice that the solid curves A and B have identical slopes of near unity for log $t<-10$, even though images (a) and (b) have sharply distinct smoothness properties. This confirms the observation in Remark 1 that the slope of the $\Gamma$ line is a finite dimensionality artifact, and is not connected to image smoothness.

\section{Degrading and restoring images; the Lips- chitz exponent as an image metrology tool}

This section deals with synthetically degraded images, and studies the behavior of Lipschitz exponents as images are degraded and restored. All Lipschitz exponents in Figures 4 and 5 were estimated using least squares fitting of the $L^{1}$ traces on the interval $-7 \leq \log t<0$. As in Figure 3, corresponding $\Sigma$ lines were not plotted to avoid clutter. Notice that all traces in Figures 4 and 5 have identical slopes for $\log t \leq-10$, irrespective of the parent image. Once again, this is spurious $\Gamma$ line behavior that must be ignored.

Our first example, in Figure 4, involves noising and denoising the $512 \times 512$ Marilyn Monroe image. The original image in Fig. 4(a) has trace $A$ and an $L^{1}$ exponent $\alpha=0.591$. Salt and pepper noise with density 0.1 was added to Fig. 4(a). This produced Fig. 4(b) with trace $B$. There is a very sharp difference between traces $A$ and $B$, and the Lipschitz exponent in Fig. 4(b) has a substantially smaller value, $\alpha=0.302$. Obviously, the addition of noise can significantly lower Lipschitz exponents, and this leads to two important 
observations. First, as mentioned in the introduction, the Lipschitz exponent reflects true image smoothness only when the image is relatively noise free. Secondly, the sensitivity of $\alpha$ to the presence of noise may be used to monitor and detect noise in imaging systems, by periodically reevaluating $\alpha$ for a suitable test image.

We now consider two distinct denoising methods. The first method used was the 'total variation' method. ${ }^{1}$ This method assumes that the original Marilyn Monroe image is of class $B V\left(R^{2}\right)$, and it is based on nonlinear partial differential equations. The Marquina-Osher scheme ${ }^{18}$ was used with $\Delta t=0.1(\Delta x)^{2}, \beta=0.0001$, and regularization parameter $\lambda=2.0$. Forward integration was pursued for 300 time steps $\Delta t$. This resulted in Fig. 4(c) and the $L^{1}$ Gaussian trace $C$. Evidently, the total variation method has eliminated a good deal of texture along with the noise in Fig. 4(c), and trace $C$ lies well below the original trace $A$. Fig. 5(c) has an $L^{1}$ Lipschitz exponent $\alpha=0.714$, larger than the original value of 0.591 . The second denoising method used was $2 \mathrm{D}$ median filtering with a $3 \times 3$ neighborhood. This produced Fig. 4(d) and $L^{1}$ trace D. Fig. 4(d) is a good approximation to the original, and trace $D$ is closer to trace $A$ than is trace $C$. The $L^{1}$ Lipschitz exponent in Fig. 4(d) is $\alpha=0.645$.

Our second example, summarized in Figure 5, involves blurring and deblurring the Marilyn Monroe image. Fig. 5(a) results from synthetic blurring of Fig. 4(a) by convolution with a Lorentzian density. The solid curve $A$ in Figure 5 is the $L^{1}$ Gaussian trace for that blurred image, and it has $\alpha=0.887$. Blurring without adding noise increases $\alpha$, as blurring is generally a smoothing operation.

Three mathematically distinct methods of deblurring Fig. 5(a) are examined under the present ideal conditions of perfect knowledge of the point spread function (psf) and no added noise. Such a study highlights the intrinsic reconstructive ability of each scheme, and the accompanying changes in $\alpha$ are of interest. The first method used was again the Marquina-Osher total variation partial differential equation procedure. ${ }^{18}$ Here, we used $\Delta t=$ $0.1(\Delta x)^{2}, \beta=0.0001$, and regularization parameter $\lambda=1000$. Forward integration was pursued for 100 time steps $\Delta t$. This resulted in Fig. 5(b) and the $L^{1}$ Gaussian trace $B$. The second method used was the Lucy-Richardson iterative procedure. ${ }^{19}$ That procedure was terminated after 200 iterations, resulting in Fig. 5(c) and trace $C$. The third method used was the "slow evolution from the continuation boundary" (SECB) procedure, ${ }^{19}$ with $s=0.01$ 
and $K=1000$. This produced Fig. $5(\mathrm{~d})$ and trace $D$.

In Fig. $5(\mathrm{~b})$, the $B V\left(R^{2}\right)$ assumption underlying the Marquina-Osher method has resulted in a smoothed out reconstruction of the original. This is particularly evident in the poorly resolved hair. As a result, the $L^{1}$ Lipschitz exponent from trace $B$ has the value $\alpha=0.695$, higher than the original value of 0.591. In Fig. 5(c), the Lucy-Richardson method is a Bayesian approach based on maximum likelihood. That method does not presume a-priori smoothness in the unknown original image. On the other hand, the Lucy-Richardson method typically requires large numbers of iterations $(>2000)$ to reconstruct fine detail. ${ }^{19}$ Terminating the procedure after 200 iterations, as is quite commonly done, and was done in Fig. 5(c), produces a smoothed out result. Nevertheless, Fig. 5(c) appears more life-like than Fig. 5(b), and the hair is better resolved. Trace $C$ almost coincides with trace $B$ for $\log t>-6$, but falls below trace $B$ for smaller $t$. Surprisingly, the Lipschitz exponent in Fig. 5(c), with a value $\alpha=0.714$, is slightly higher than in Fig. 5(b). The SECB procedure in Fig. 5(d) is a noniterative direct deblurring method, based on solving an ill-posed fractional diffusion equation backwards in time. The "slow evolution" constraint ${ }^{19}$ that is used to regularize that problem does not require smoothness, and allows for non smooth solutions not in $B V\left(R^{2}\right)$. For this reason, Fig. $5(\mathrm{~d})$ appears to be a higher quality approximation to the original image than the previous two reconstructions. This is reflected in trace $D$ which lies above traces $B$ and $C$ and has a well-defined elbow. The $L^{1}$ Lipschitz exponent in Fig. 5(d) has the value $\alpha=0.616$, quite close to the original value of 0.591 .

\section{Real imagery, APEX blind deconvolution, and Lipschitz exponents}

The above experiments on synthetically degraded imagery with perfectly known psfs are instructive, and they indicate the Lipschitz exponent to be a useful image metrology tool. However, in many imaging situations that result in blurred imagery, the system psf is generally only poorly known. Effective methods of "blind deconvolution" that do not require knowledge of the psf are of considerable interest, but it must be emphasized that such procedures are fraught with serious mathematical and computational difficulties regarding 
uniqueness and convergence.

The APEX method is a recently developed blind deconvolution technique that is targeted at a specific class of shift-invariant blurs, in the form of $2 \mathrm{D}$ isotropic, bell-shaped, heavy-tailed, probability density functions. Not all images can be usefully enhanced with the APEX method, but the method has been found useful in a variety of applications, including astronomy, nanoscale electron microscopy, and MRI and PET brain imaging. ${ }^{6,20,21}$ As a rule, APEX-detected psfs that successfully sharpen these images turn out to be low exponent Lévy stable laws. ${ }^{6,20,21}$ Such blurs are very far from Gaussians or Lorentzians, and are not generally known in the imaging literature.

A-priori knowledge about the solution is a fundamental aspect of solving inverse problems in applied mathematics. This is especially the case with blind deconvolution where severe ill-conditioning is compounded with non uniqueness of solutions. The plausibility of APEX reconstructions must be gauged by experienced analysts using independent considerations. Successful APEX deconvolution should produce an image that is visibly sharper than the original, yet one where the newly enhanced features can be traced back to the original. Such sharpening should be accompanied by a measurable drop in Lipschitz exponent. A decrease in Lipschitz exponent reflects the extent to which small scale information has been reconstructed. Quantitative confirmation of sharpening is one of several elements that bear on the plausibility of the APEX image. We now give several examples of Lipschitz behavior before and after APEX processing. These examples involve real images originating from significant areas of application.

Our first example, in Fig. 6, involves an iconic Hubble Space Telescope Whirlpool galaxy (M51) image. The original full resolution image was taken with NASA's ultra sophisticated Advanced Camera for Surveys (ACS). APEX processing of the stepped down $710 \times 1024$ color image is discussed in detail in Ref. 21. Here, we examine Lipschitz behavior in the blue component of that image, before and after APEX processing. Using least squares fitting on the interval $-11 \leq \log t<0$, we find that the original image 6 (a) has the $L^{1}$ Gaussian trace $A$ with Lipschitz exponent $\alpha=0.416$. The APEX image 6 (b) again shows substantial additional detail, and it has the $L^{1}$ Gaussian trace $B$ with Lipschitz exponent $\alpha=0.191$. This is a remarkable $54 \%$ drop in $\alpha$.

We next consider brain imaging. APEX processing of the $512 \times 512$ sagittal MRI brain image in Fig. 7 was previously discussed in Ref. 6 . 
Using least squares fitting on the interval $-7 \leq \log t<0$, we find that the original MRI image 7(a) has the $L^{1}$ Gaussian trace $A$ with Lipschitz exponent $\alpha=0.447$. The APEX image 7(b) is significantly sharper visually. It has the $L^{1}$ Gaussian trace $B$ with Lipschitz exponent $\alpha=0.296$. This represents a $34 \%$ decrease in $\alpha$.

In functional PET imaging, a positron emitting radionuclide is injected into the patient, and used to tag glucose molecules in their course through the brain. Performing specific mental tasks activates various parts of the brain, causing increased glucose uptake and increased positron emission. Centers of activity translate into relatively bright spots in the PET image. However, blurring tends to attenuate such relative differences, resulting in a loss of contrast. APEX processing of the transverse PET image in Fig. 8 was previously discussed in Ref. 6 . The $512 \times 512$ image in Fig. 8(a) is a stepped up version of an original $128 \times 128$ image. Using least squares fitting on the interval $-6 \leq \log t<0$, we find that image 8 (a) has the $L^{1}$ Gaussian trace $A$ with Lipschitz exponent $\alpha=0.502$. The APEX-processed image $8(\mathrm{~b})$ shows identical features as in 8(a), but contrast has been substantially increased. Significantly, several bright spots appear in $8(\mathrm{~b})$ that were not apparent in 8(a). Image $8(\mathrm{~b})$ has the $L^{1}$ Gaussian trace $B$ with Lipschitz exponent $\alpha=0.394$. This is a $21 \%$ decrease in $\alpha$. The low resolution initial $128 \times 128$ PET format is a serious limitation in this example. Higher resolution PET imagery would enable more substantial APEX sharpening.

The last example involves state of the art nanoscale SEM. Previous work on applying APEX blind deconvolution to SEM imagery is discussed in Ref. 20. Since that time, significantly more powerful equipment has become available. $^{\dagger}$ It is an interesting fact that the high quality imagery produced by this new instrumentation can still be usefully enhanced by the APEX method. Moreover, Lipschitz analysis can be used to confirm such sharpening.

Fig. 9 deals with a $\mathrm{Au} / \mathrm{Pd}$ decorated magnetic tape sample, taken with a Hitachi S-4800 SEM using an accelerating voltage of $1 \mathrm{kV}$. The field of view is $1 \mu \mathrm{m}$. The original $1024 \times 768$ micrograph in 9 (a) has the $L^{1}$ Gaussian trace $A$ with Lipschitz exponent $\alpha=0.303$. The APEX-processed micrograph 9(b),

\footnotetext{
${ }^{\dagger}$ Certain commercial equipment is identified in this paper to adequately describe experimental procedures. Such identification does not imply recommendation or endorsement by the National Institute of Standards and Technology, nor does it imply that the equipment so identified is necessarily the best available for the purpose.
} 
which is noticeably sharper, has the $L^{1}$ Gaussian trace $B$ with Lipschitz exponent $\alpha=0.234$. This is a $23 \%$ decrease in $\alpha$. Least squares fitting on the interval $-7 \leq \log t<0$ was used.

Sharpening in Figs. 9(b) was confirmed independently, using a proprietary FEI software package.

Remark 3: Uncertainty in Lipschitz exponents. Estimated Lipschitz exponent values in all examples in this paper, depend on the choice that was made for the 'slowly varying' interval in the trace of $\mu(t)$, as discussed in Section 5. Least squares fitting on that interval produces the $\Sigma$ line whose slope is $\alpha / 2$. The particular choice of interval is a matter of judgment. A somewhat larger or smaller interval might have been used in any given case, resulting in a slightly different value for $\alpha$. However, as was always the case here, when Lipschitz exponents are used to evaluate the effectiveness of an image processing algorithm on a given image, the same fitting interval should be used in 'before and after' comparisons.

\section{Conclusion}

A mathematical framework for quantifying the unsmoothness of images, leading to an effective computational tool for estimating image Lipschitz exponents, has been presented. The $L^{1}$ Lipschitz exponent $\alpha$, where $0<\alpha \leq 1$, is a useful image metrology tool that measures fine scale content, provided the image is relatively noise free. It is found that natural images have $L^{1}$ values of $\alpha$ lying between 0.2 and 0.5 , typically. Such images are not of bounded variation, since this requires $\alpha=1.0$.

The use of Lipschitz exponents in the quantitative evaluation of image reconstruction procedures was studied. Using synthetically degraded images, it was shown that Lipschitz exponents can measure the extent to which competing denoising algorithms remove texture along with the noise. Likewise, Lipschitz exponents can measure the ability of competing deblurring algorithms to recover texture. Quantitative confirmation of fine structure recovery is particularly important in blind image deconvolution, where the cause of the blur is unknown. This was illustrated in the case APEX blind deconvolution applied to Hubble space telescope imagery, scanning electron microscopy, and MRI and PET brain scans. 


\section{References}

[1] L. Rudin, S. Osher, and E. Fatemi, "Nonlinear total variation based noise removal algorithms," Physica D. 60, 259-268 (1992).

[2] G. Aubert and P. Kornprobst, Mathematical Problems in Image Processing, Springer, New York, 2002.

[3] V. Caselles, J. M. Morel, G. Sapiro, and A. Tannenbaum Eds., Special Issue on Partial Differential Equations and Geometry-Driven Diffusion in Image Processing, IEEE Transactions on Image Processing 7, March 1998.

[4] J. Weickert, Anisotropic Diffusion in Image Processing, B. G. Teubner, Stuttgart, Germany 1998.

[5] Y. Gousseau and J. M. Morel, "Are natural images of bounded variation ?" SIAM J. Math. Anal. 33, 634-648 (2001).

[6] A. S. Carasso, "The APEX method in image sharpening and the use of low exponent Lévy stable laws", SIAM J. Appl. Math. 63, 593-618 (2002).

[7] A. S. Carasso, "Singular integrals, image smoothness, and the recovery of texture in image deblurring," SIAM J. Appl. Math. 64, 1749-1774 (2004).

[8] A. E. Vladár, M. T. Postek, and M. P. Davidson, "Image sharpness measurement in scanning electron microscopy", Scanning 20, pp. 24-34 (1998).

[9] M. H. Taibleson, "On the theory of Lipschitz spaces of distributions on Euclidean n-space. I. Principal properties", Journal of Mathematics and Mechanics 13, 407-478 ( 1964).

[10] P. L. Butzer and R. J. Nessel, Fourier Analysis and Approximation, Academic Press, New York, 1971.

[11] J. W. Goodman, Introduction to Fourier Optics, Mc Graw-Hill, New York, 1968. 
[12] J. D. Gaskill, Linear Systems, Fourier Transforms, and Optics, Wiley, New York, 1978.

[13] H. C. Andrews and B. R. Hunt, Digital Image Restoration, Prentice Hall, Englewood Cliffs, NJ 1977.

[14] W. K. Pratt, Digital Image Processing, Second ed., Wiley, New York, 1991.

[15] R. C. Gonzalez and P. Wintz, Digital Image Processing, Second ed., Addison-Wesley, Reading, MA 1987.

[16] W. Feller, An Introduction to Probability Theory and its Applications, Vol. 2, Second ed., Wiley, New York, 1971.

[17] E. O. Brigham, The Fast Fourier Transform and its Applications, Prentice-Hall, Englewood Cliffs, New Jersey, 1992.

[18] A. Marquina and S. Osher, "Explicit algorithms for a new time dependent model based on level set motion for nonlinear deblurring and noise removal", SIAM J. Sci. Comput. 22, 387-405 (2000).

[19] A. S. Carasso, "Linear and nonlinear image deblurring: a documented study", SIAM J. Numer. Anal. 36, 1659-1689 (1999).

[20] A. S. Carasso, D. S. Bright, and A. E. Vladár, "The APEX method and real-time blind deconvolution of scanning electron microscope imagery", Optical Engineering 41, 2499-2514 (2002).

[21] A. S. Carasso, "APEX blind deconvolution of color Hubble space telescope imagery and other astronomical data", Optical Engineering 45, 107004 (2006). 


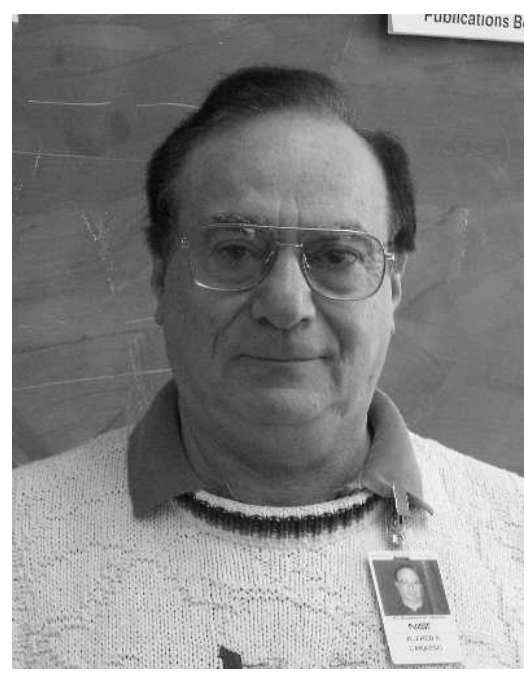

Biography Alfred S. Carasso received the Ph.D degree in mathematics at the University of Wisconsin in 1968. He was a professor of mathematics at the University of New Mexico, and a visiting staff member at the Los Alamos National Laboratory, prior to joining the National Institute of Standards and Technology in 1982. His major research interests lie in the theoretical and computational analyses of ill-posed problems in partial differential equations, together with their application in image reconstruction and computer vision. He is the author of original theoretical papers, is a patentee in the field of image processing, and is an active speaker at national and international conferences in applied mathematics. 


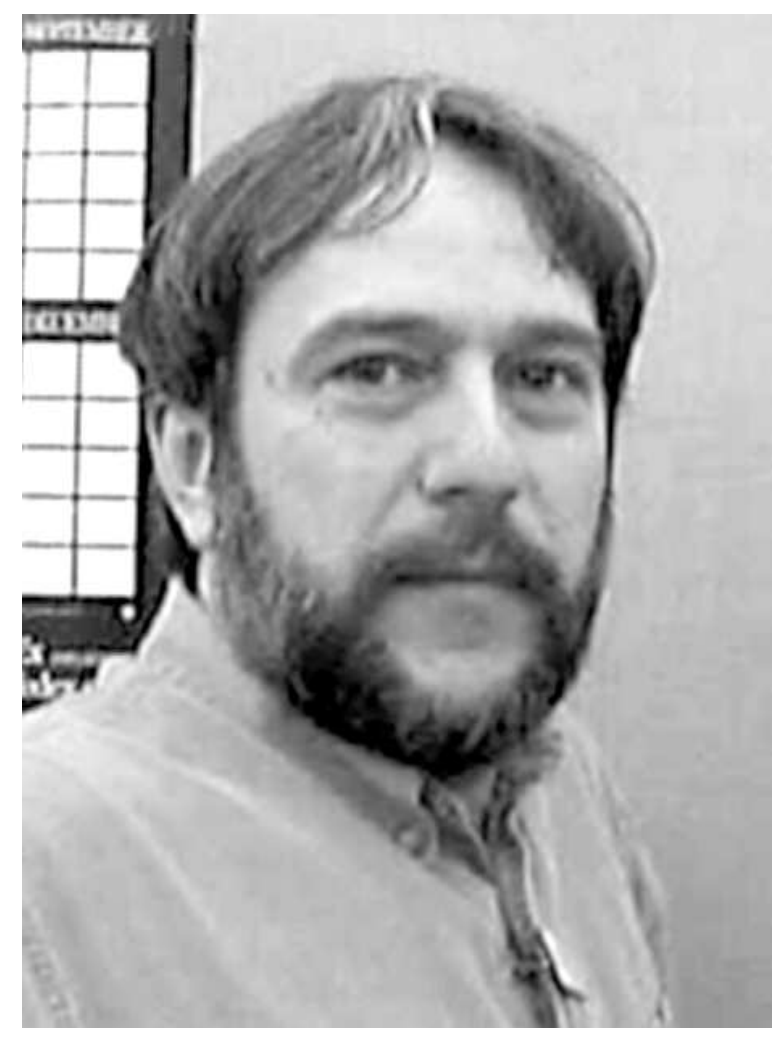

Biography Dr. András E. Vladár is the leader of the Scanning Electron Microscope Metrology Project in the Nanoscale Metrology Group at the National Institute of Standards and Technology. He holds M.S. (1977) and Ph.D. (1984) degrees in Electrical Engineering from the Technical University of Budapest, Hungary. Until 1991 he worked as a Research Fellow at the Research Institute for Technical Physics of the Hungarian Academy of Sciences, Budapest. Dr. Vladár is an expert in scanning electron microscope (SEM) critical dimension (CD) metrology; he has developed several metrology systems based on SEMs. Prior to joining NIST in 1999, he was a member of the technical staff at the HewlettPackard ULSI Research Laboratory and primarily worked on accurate, fast, and effective dimensional measurement methods for $100 \mathrm{~nm}$ silicon integrated circuit technology. At HewlettPackard he established sound metrology methods, procedures and practices that significantly improved the speed and quality of dimensional measurements, and surface inspection and film thickness 
measurements in the Class 1 integrated circuit fabrication facility. He identified the main sources of measurement errors and developed several successful methods to eliminate many of them. 


\section{List of Figure Captions}

Fig. 1. $512 \times 512$ Giza Pyramids image is not of bounded variation, but has an $L^{1}$ Lipschitz exponent $\alpha \approx 0.32$. This follows from the graphical use of Theorem 1, using the FFT techniques discussed in Section 4. Solid curve $\mathrm{A}$ is a plot of $\mu(t)=\left\|G_{*}^{t} f-f\right\|_{1} /\|f\|_{1}$ versus $t$, on a log-log scale, where $G_{*}^{t} f$ is defined in Eq.(14). Majorizing dashed straight line $\Gamma$, defined by $\log \mu(t)=7.72+0.988 \log t$, accurately captures linear behavior in Eq. (17) for very small values of $t$, but is grossly inaccurate at larger values of $t$. Linear behavior at very small $t$ is misleading, and is unrelated to true image smoothness. (See Remark 1). Majorizing dashed straight line $\Sigma$, defined by $\log \mu(t)=-1.31+0.159 \log t$, accurately reflects behavior for $-10 \leq \log t \leq-1$, while being grossly inaccurate at very small $t$. Behavior along $\Sigma$ is taken to be true behavior in Giza Pyramids image, and indicates $\left\|G_{*}^{t} f-f\right\|_{1} \leq 0.27\|f\|_{1} t^{0.159}, 0<t \leq 0.1$. From Eq. (9), this implies image $\in \Lambda(0.318,1, \infty)$.

Fig. 2. The above $512 \times 5128$-bit images have $L^{1}$ Lipschitz exponents $\alpha$ in the range $0.18<\alpha<0.54$, and are not of bounded variation. See Table 1 .

Fig. 3. Galaxy clusters can have very low Lipschitz exponents. Above images of Abell 426 (Perseus) cluster obtained from NASA STScI Digitized Sky Survey. Images (a) and (b) are of size $512 \times 512$ and each is centered on the exact same point in the sky. Image (a) covers a $1 / 2 \mathrm{Mpc}$ square of the sky, while image (b) covers a $1 \mathrm{Mpc}$ square. Solid curves A and B are the corresponding plots of $\mu(t)=\left\|G_{*}^{t} f-f\right\|_{1} /\|f\|_{1}$ versus $t$, on log-log scale. Using least squares fitting on $-6 \leq \log t \leq 0$, we find that image (a) has an $L^{1}$ Lipschitz exponent $\alpha=0.116$, while image (b) has $\alpha=0.042$. Corresponding $\Sigma$ lines were not plotted to avoid clutter. Solid curves A and $\mathrm{B}$ have identical slopes $\approx 1$ for $\log t<-10$. This confirms the observation in Remark 1 that the slope of the $\Gamma$ line is a finite dimensionality artifact, and is not connected to image smoothness.

Fig. 4. Image Denoising. Noise addition can artificially lower Lipschitz exponent $\alpha$, while some noise removal algorithms can eliminate texture and 
increase $\alpha$. Gaussian traces A, B, C, and D correspond to images (a), (b), (c), and $(d)$ respectively. Least squares fitting on $(-7,0)$ produced the following results. ( $\Sigma$ lines not plotted to avoid clutter). (a) Original Marilyn Monroe image has $L^{1}$ exponent $\alpha=0.591$. (b) Adding salt and pepper noise to image (a) leads to $\alpha=0.302$, a very noticeable reduction. (c) Nonlinear partial differential equation 'total variation' noise removal algorithm eliminates noise, as well as texture, and results in $\alpha=0.714$, higher than in original. (d) 2D median filtering produces closer approximation to original, with $\alpha=0.645$.

Fig. 5. Image Deblurring. Not all deblurring algorithms are equally effective. Gaussian traces A, B, C, and D correspond to images (a), (b), (c), and $(\mathrm{d})$ respectively. Least squares fitting on $(-7,0)$ produced the following results. ( $\Sigma$ lines not plotted to avoid clutter). (a) Syntheticaly blurred Marilyn Monroe image has $L^{1}$ exponent $\alpha=0.887$. (b) Nonlinear partial differential equation 'total variation' deblurring of (a) erodes texture and leads to $\alpha=0.695$. (c) Lucy-Richardson deblurring has $\alpha=0.714$. (d) SECB deblurring gives $\alpha=0.616$. This is closest to original image value $\alpha=0.591$.

Fig. 6. Evaluating the effectiveness of APEX blind deconvolution of Whirlpool galaxy (M51) image. See Ref. 25. (a) Original Hubble Space Telescope $710 \times 1024$ image has Gaussian trace $A$ and $L^{1}$ Lipschitz exponent $\alpha=0.416$. (b) APEX processed image has Gaussian trace $B$ and $L^{1}$ Lipschitz exponent $\alpha=0.191$, a $54 \%$ decrease. Least squares fitting on $(-11,0)$ was used.

Fig. 7. Evaluating the effectiveness of APEX blind deconvolution of sagittal brain MRI image. See Ref. 6. (a) Original $512 \times 512$ image has Gaussian trace $A$ and $L^{1}$ Lipschitz exponent $\alpha=0.447$. (b) APEX processed image has Gaussian trace $B$ and $L^{1}$ Lipschitz exponent $\alpha=0.296$, a $34 \%$ decrease. Least squares fitting on $(-7,0)$ was used.

Fig. 8. Evaluating the effectiveness of APEX blind deconvolution of transverse functional PET brain slice. See Ref. 6. (a) Original $512 \times 512$ image has Gaussian trace $A$ and $L^{1}$ Lipschitz exponent $\alpha=0.502$. (b) APEX processed image has Gaussian trace $B$ and $L^{1}$ Lipschitz exponent $\alpha=0.394$, a $21 \%$ decrease. Least squares fitting on $(-6,0)$ was used.

Fig. 9. Evaluating the effectiveness of APEX blind deconvolution in nanoscale 
Scanning Electron Microscopy. (a) Original $1024 \times 768$ micrograph of $\mathrm{Au} / \mathrm{Pd}$ decorated magnetic tape sample has Gaussian trace $A$ and $L^{1}$ Lipschitz exponent $\alpha=0.303$. (b) APEX processed image has Gaussian trace $B$ and $L^{1}$ Lipschitz exponent $\alpha=0.234$, a $23 \%$ decrease. Least squares fitting on $(-7,0)$ was used. 
TABLE 1

Values of $\left(C_{1}, \alpha\right)$ in Eq.(18) for each image $f(x, y)$ in Figure 2.

\begin{tabular}{|c|c|c|}
\hline Image & Size & $L^{1}$ norm values of $C_{1}$ and $\alpha$ \\
\hline Sydney Opera House & $512^{2}$ & $C_{1}=0.42, \quad \alpha=0.476$ \\
\hline USS Theodore Roosevelt & $512^{2}$ & $C_{1}=0.69, \quad \alpha=0.535$ \\
\hline Washington DC Landsat & $512^{2}$ & $C_{1}=0.80, \quad \alpha=0.236$ \\
\hline Heart ventricle SPECT & $512^{2}$ & $C_{1}=0.70, \quad \alpha=0.330$ \\
\hline Transverse brain PET & $512^{2}$ & $C_{1}=1.07, \quad \alpha=0.502$ \\
\hline Sagittal brain MRI & $512^{2}$ & $C_{1}=0.83, \quad \alpha=0.447$ \\
\hline Weather satellite Hurricane & $512^{2}$ & $C_{1}=0.82, \quad \alpha=0.411$ \\
\hline Nanoscale electron micrograph & $512^{2}$ & $C_{1}=0.69, \quad \alpha=0.183$ \\
\hline USAF resolution chart & $512^{2}$ & $C_{1}=2.08, \quad \alpha=0.203$ \\
\hline
\end{tabular}


L1 RELATIVE ERROR IN GAUSSIAN APPROXIMATION

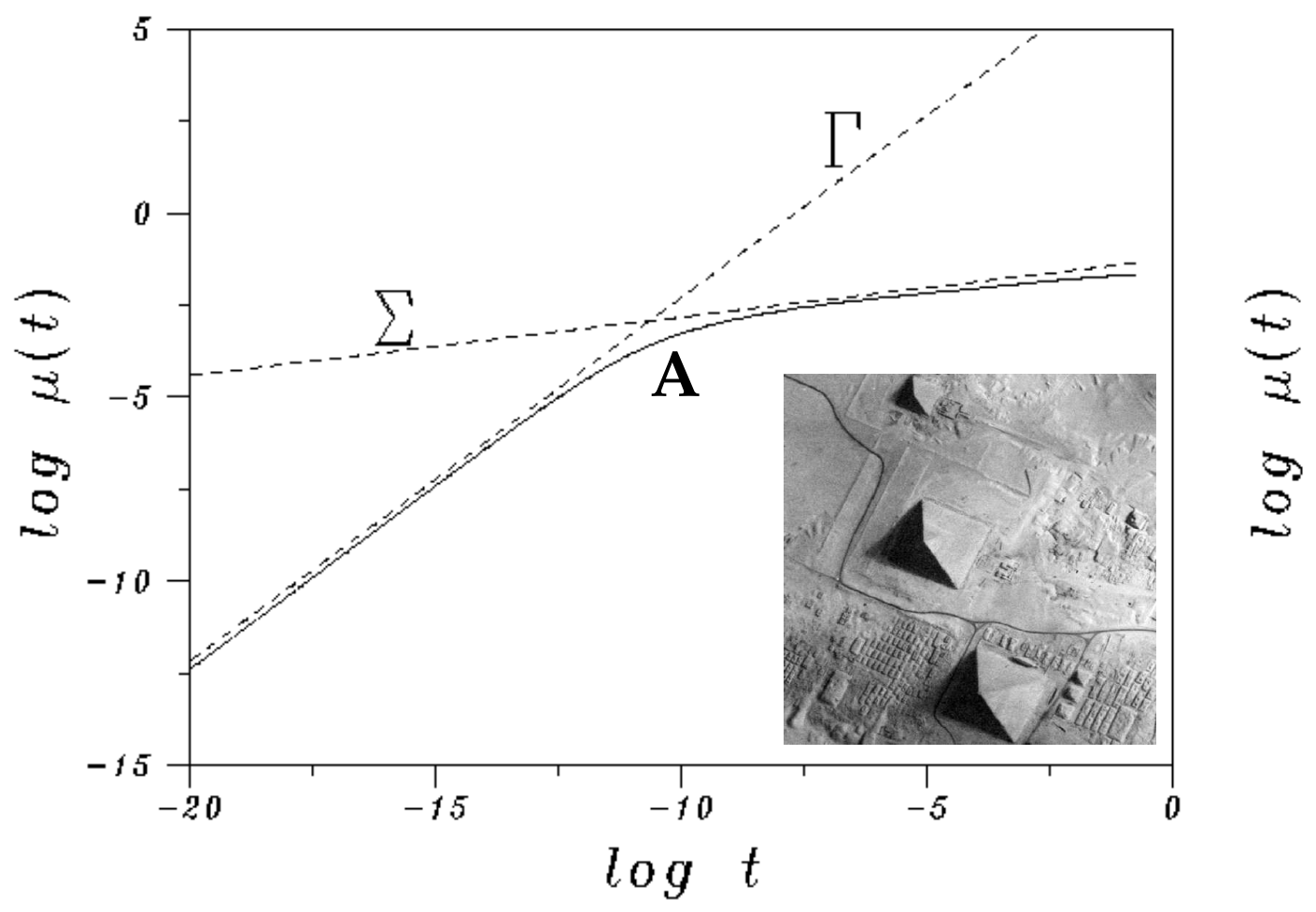

Figure 1: $512 \times 512$ Giza Pyramids image is not of bounded variation.... 

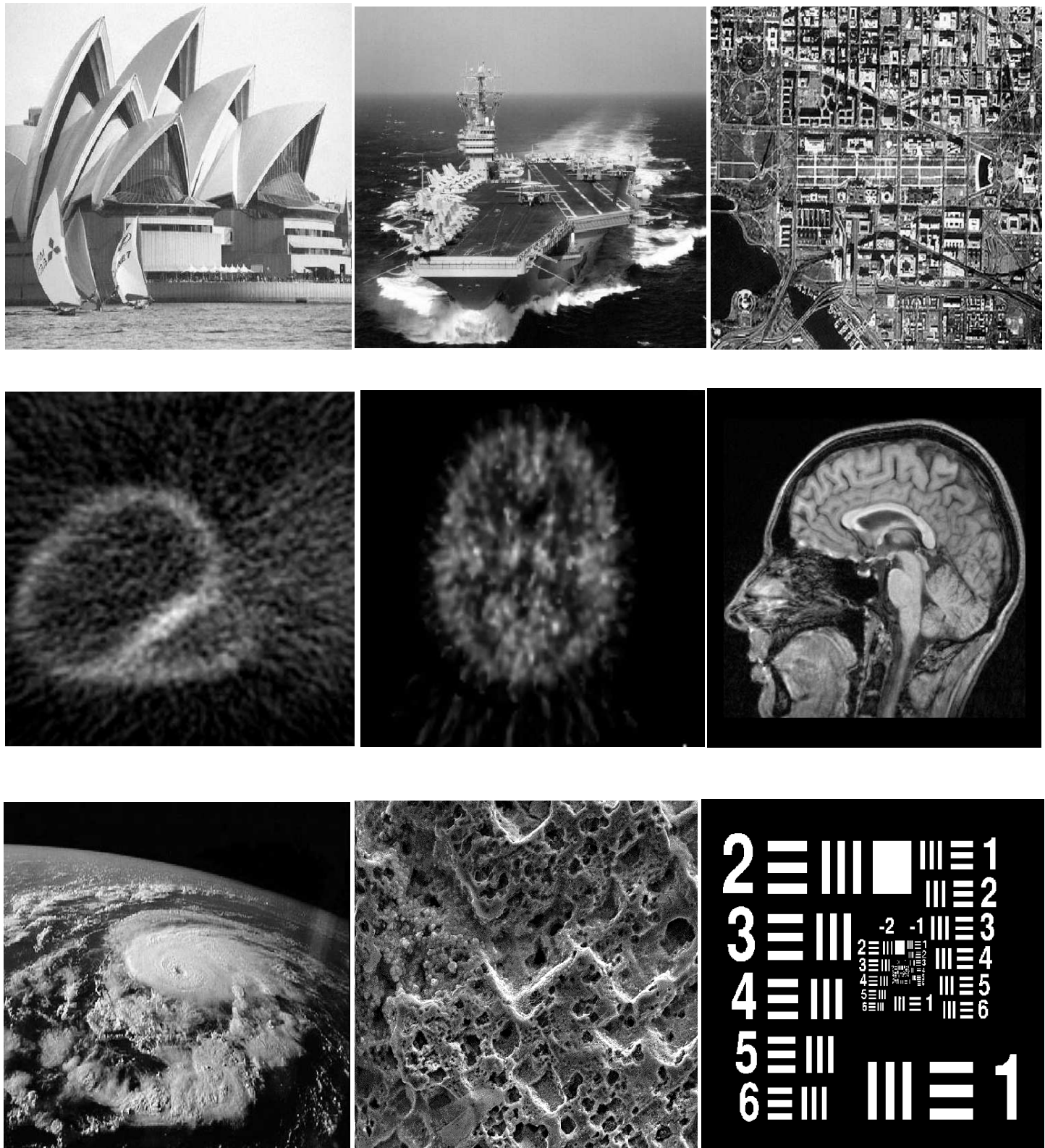

Figure 2: The above $512 \times 5128$-bit images have $L^{1}$ Lipschitz exponents...... 


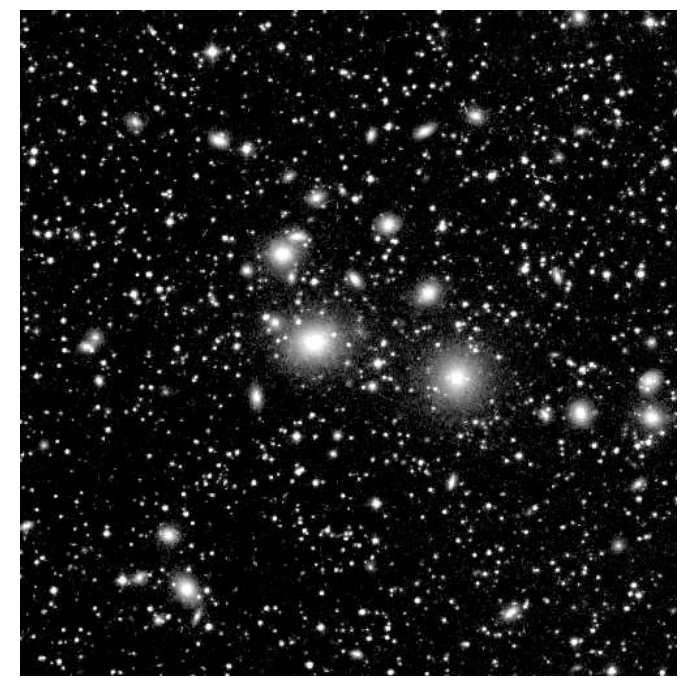

(a)

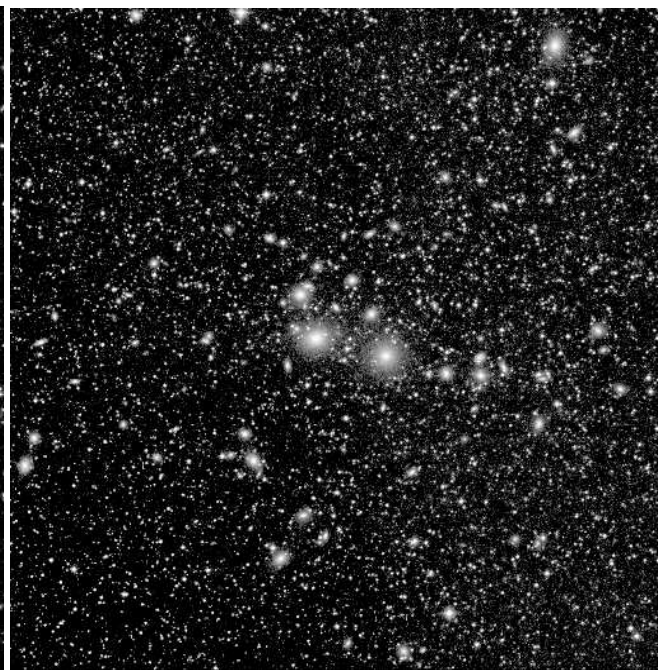

(b)

L1 RELATIVE ERROR IN GAUSSIAN APPROXIMATION

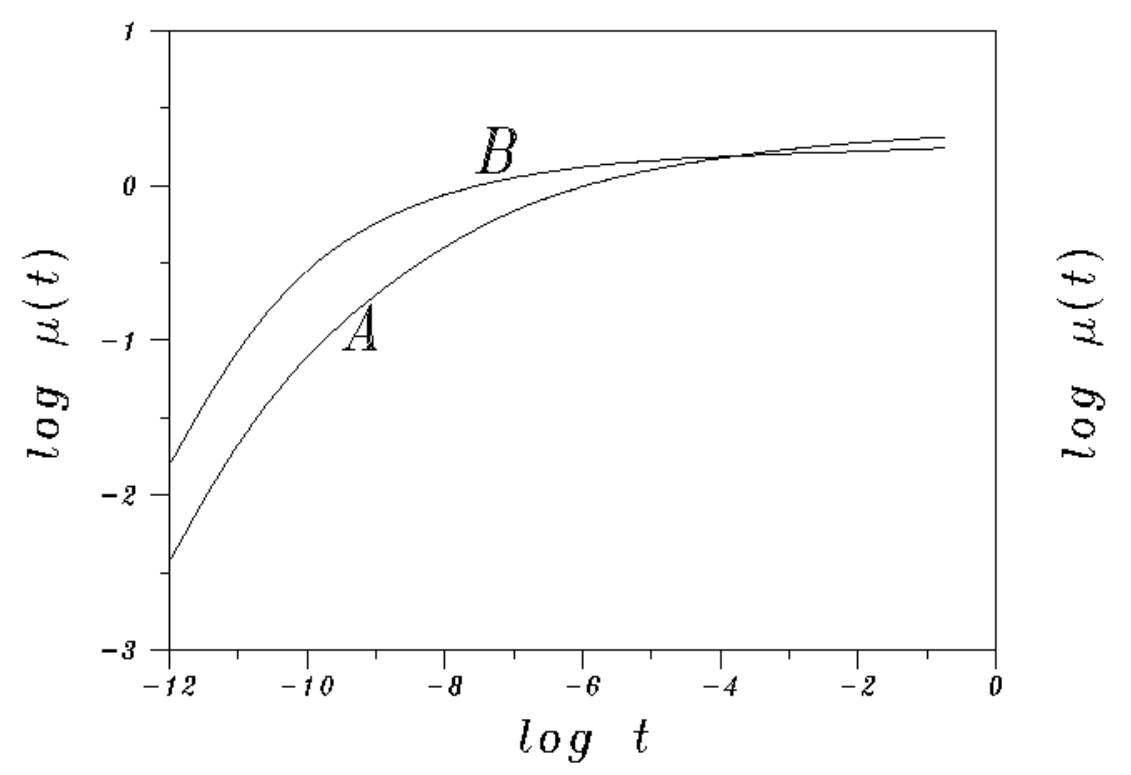

Figure 3: Galaxy clusters can have very low Lipschitz exponents..... 


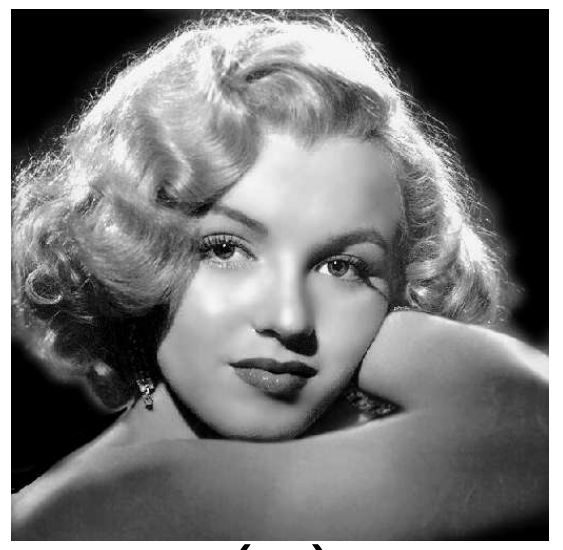

(a)

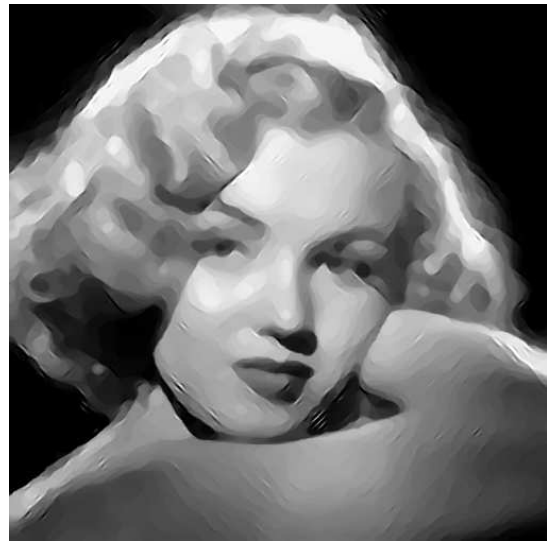

(c)

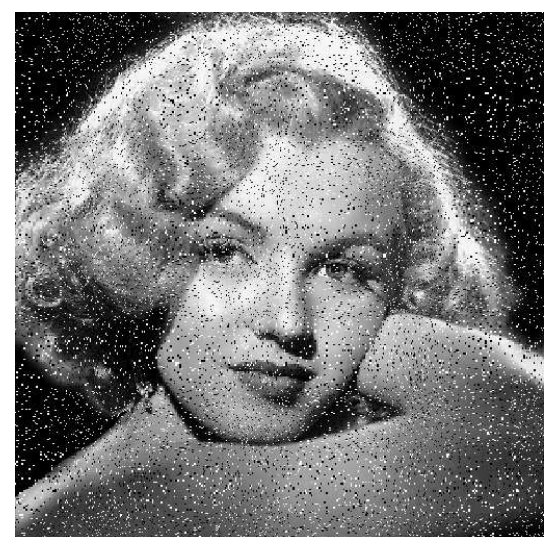

(b)

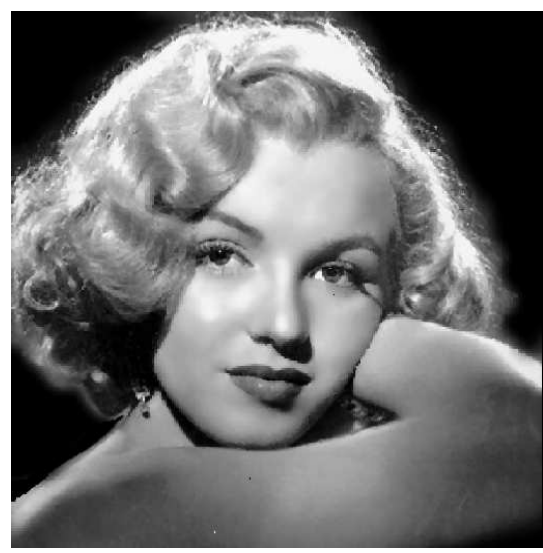

(d)

L1 RELATIVE ERROR IN GAUSSIAN APPROXIMATION

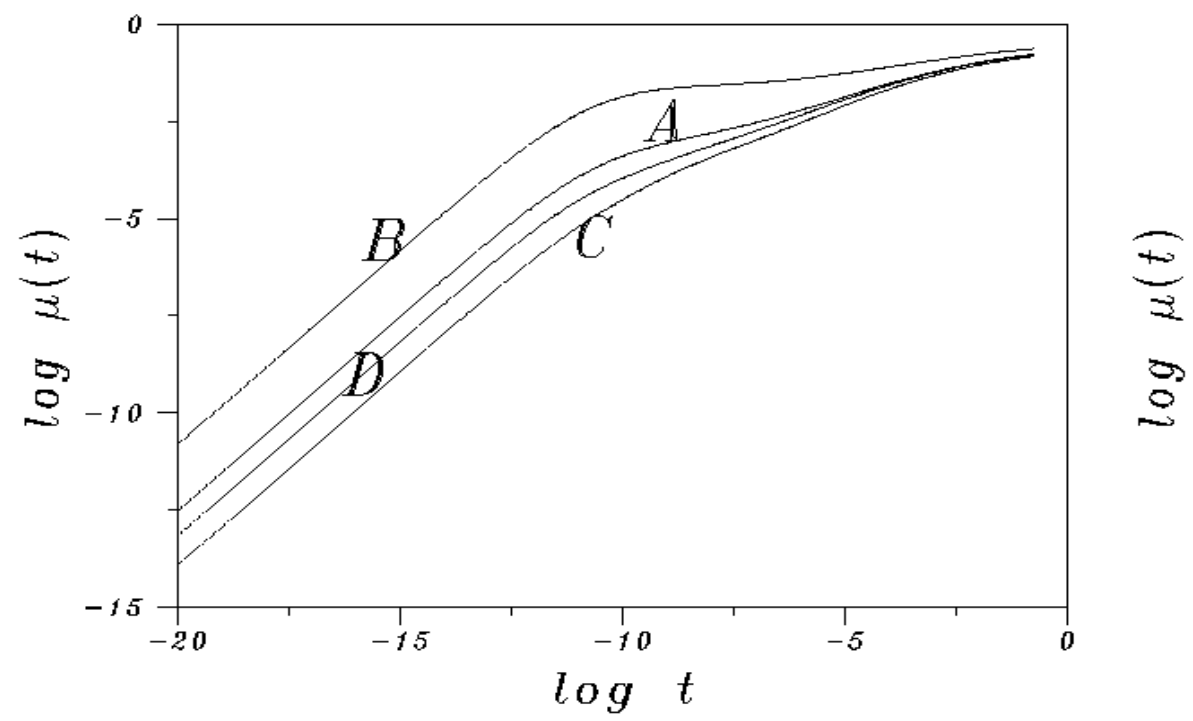

Figure 4: Image Denoising. Noise addition can artificially ....... 


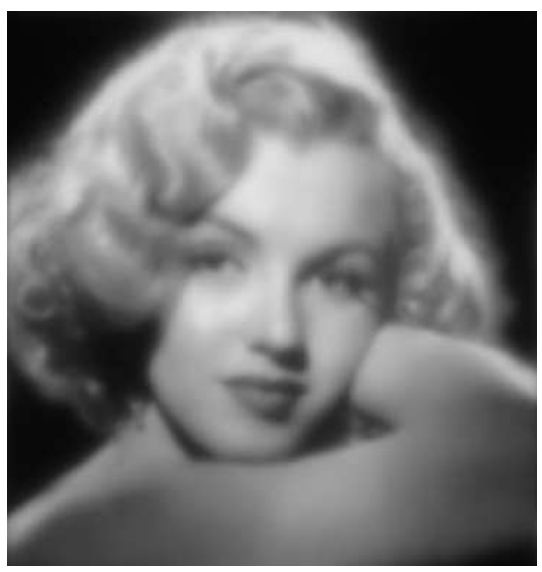

(a)

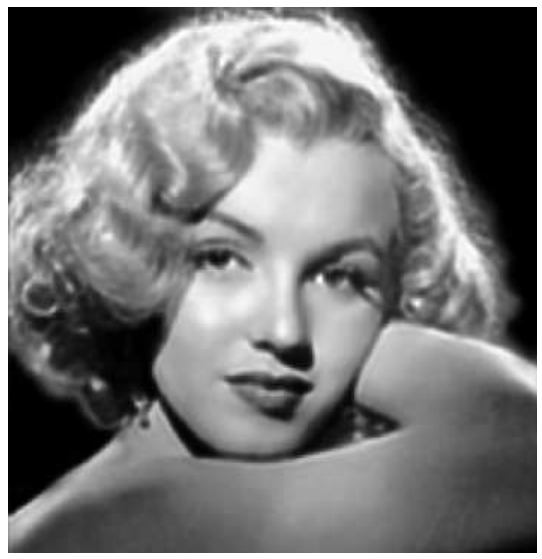

(c)

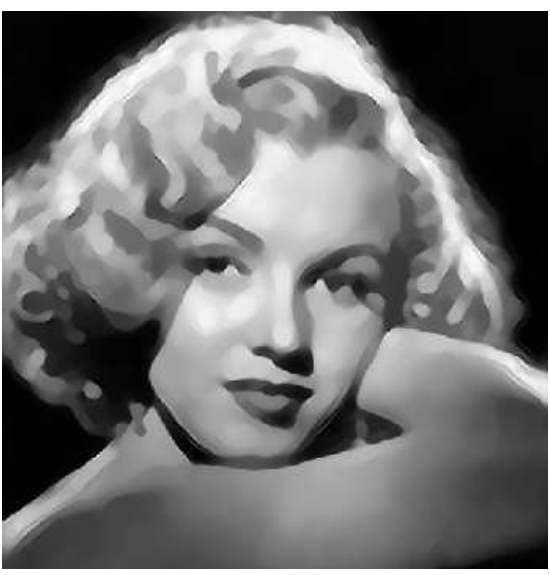

(b)

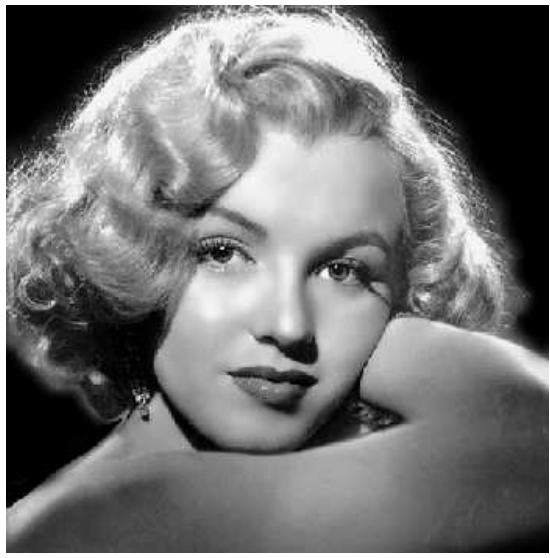

(d)

L1 RELATIVE ERROR IN GAUSSIAN APPROXIMATION

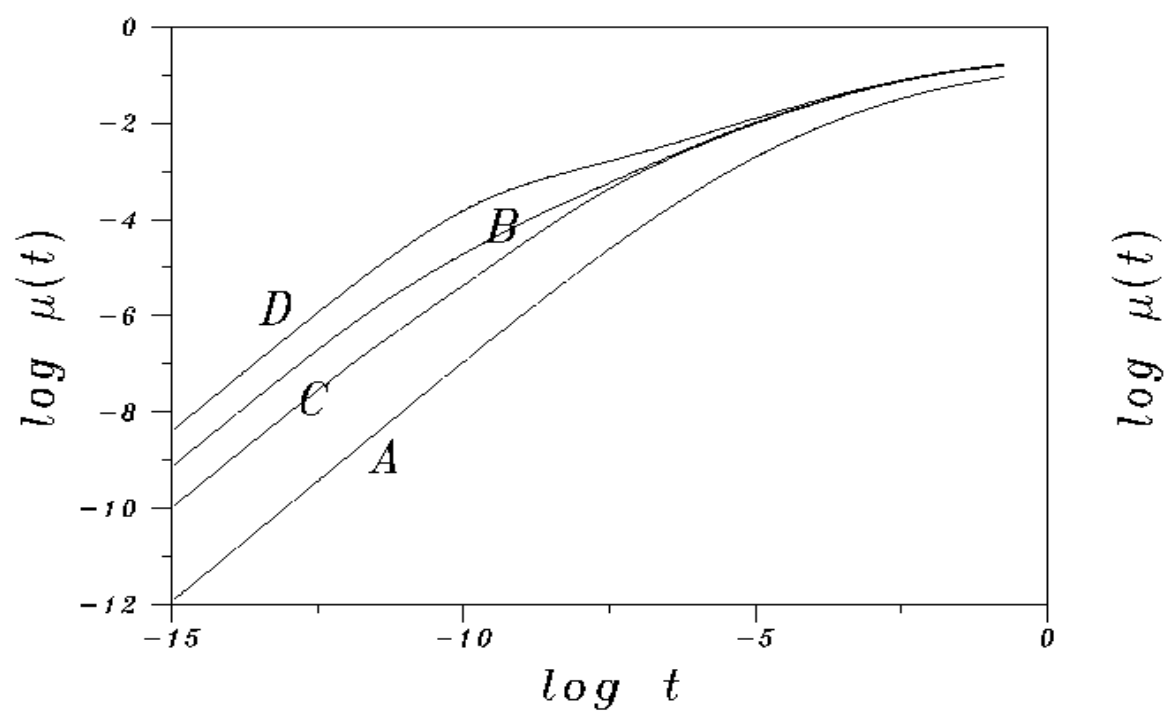

Figure 5: Image Deblurring. Not all deblurring algorithms 


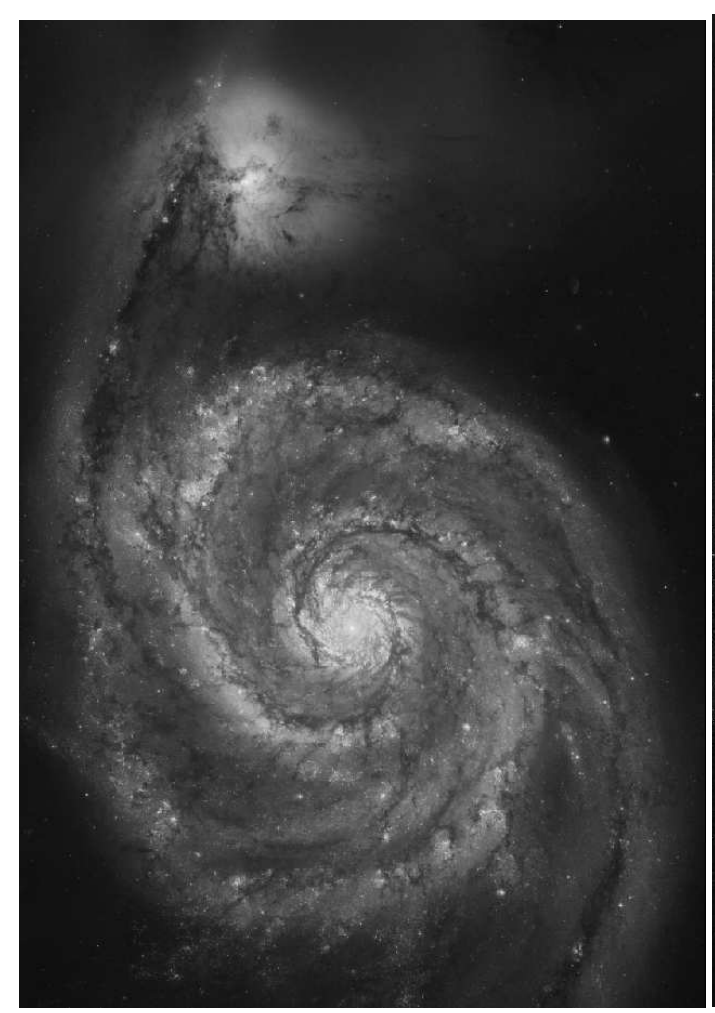

(a)

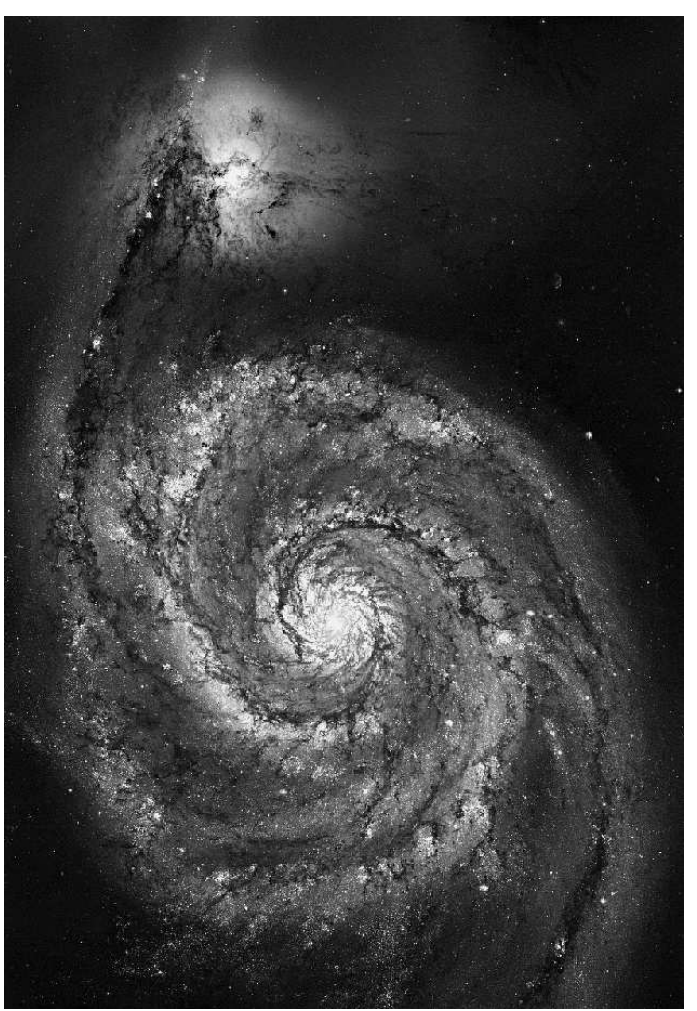

(b)

L1 RELATIVE ERROR IN GAUSSIAN APPROXIMATION

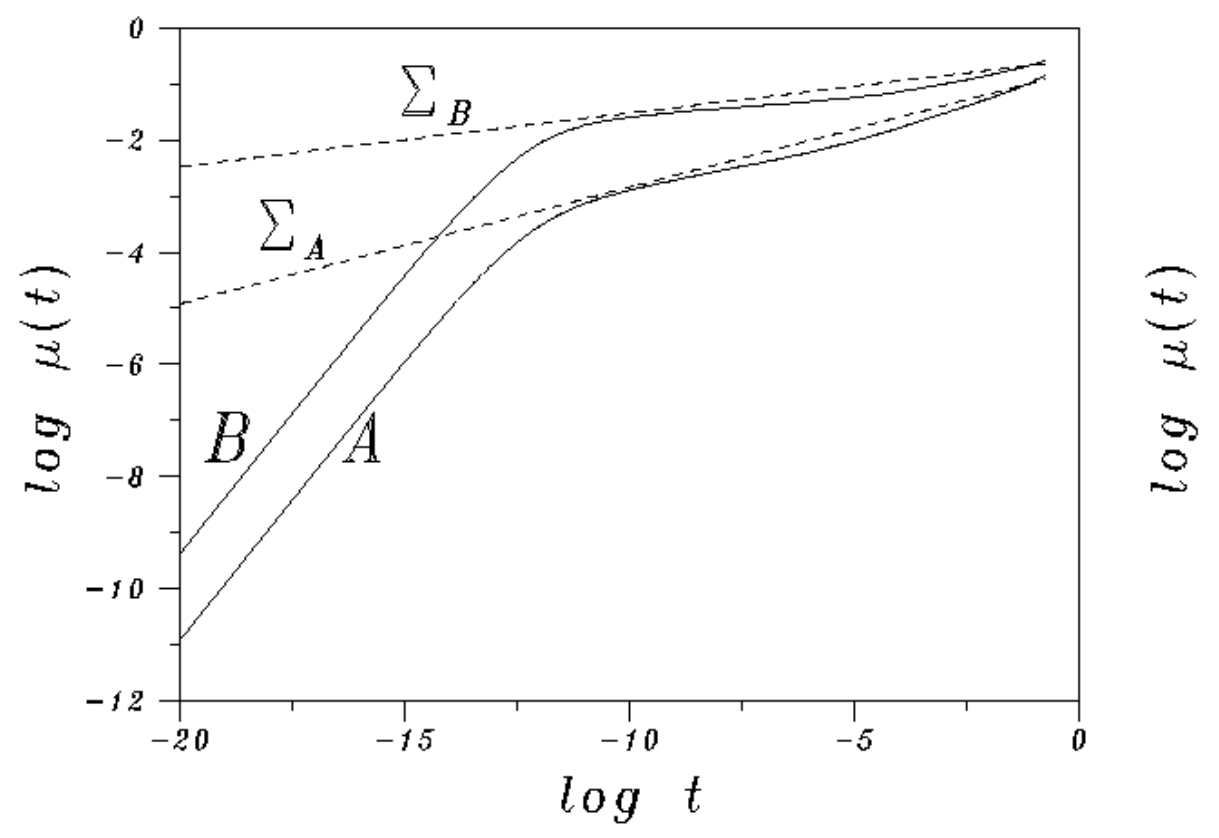

Figure 6: Evaluating the effectiveness.....Whirlpool galaxy 


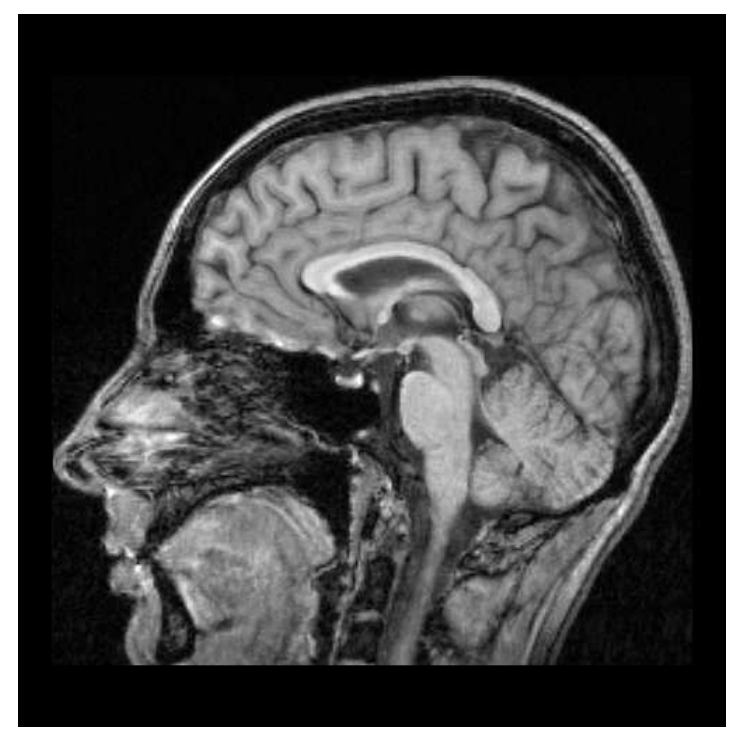

(a)

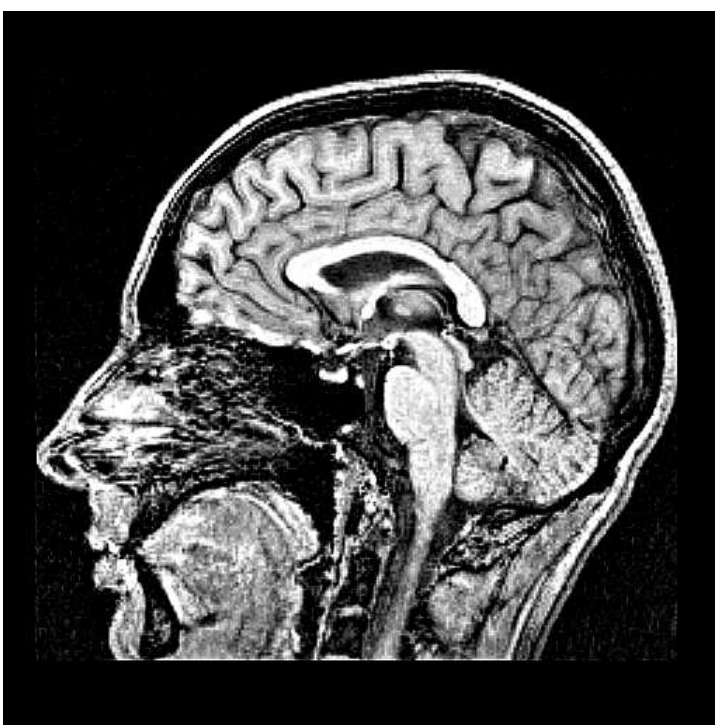

(b)

L1 RELATIVE ERROR IN GAUSSIAN APPROXIMATION

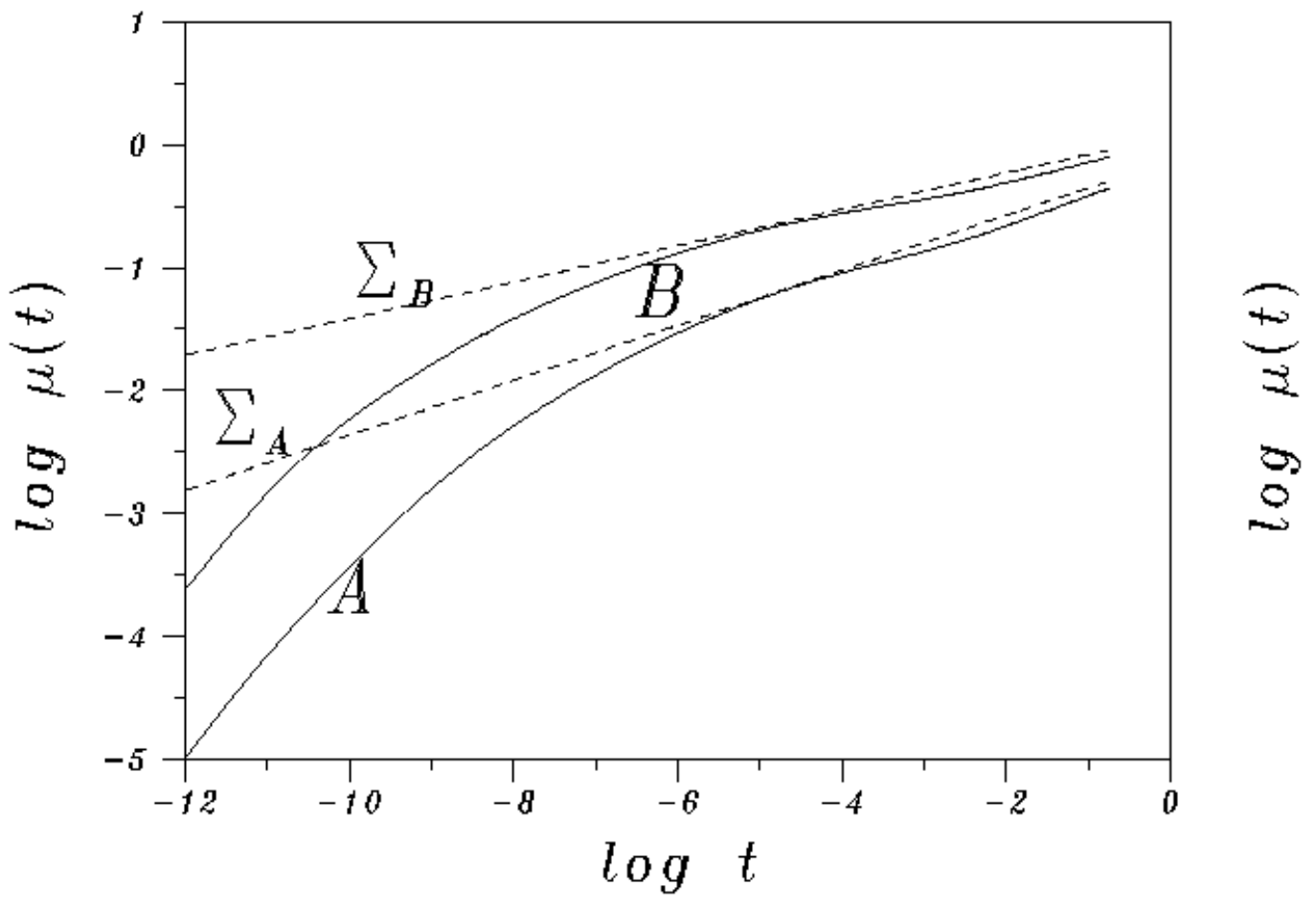

Figure 7: Evaluating the effectiveness......sagittal brain MRI .... 


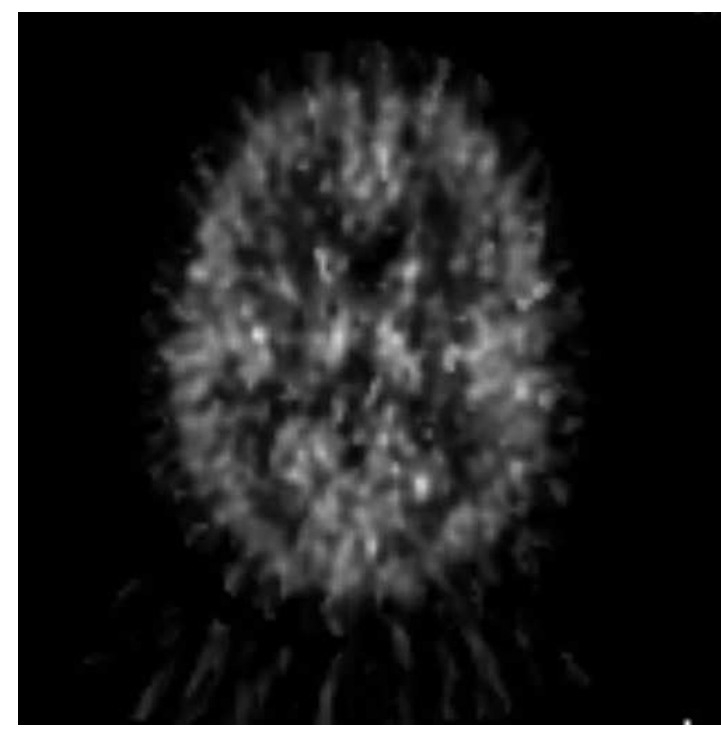

(a)

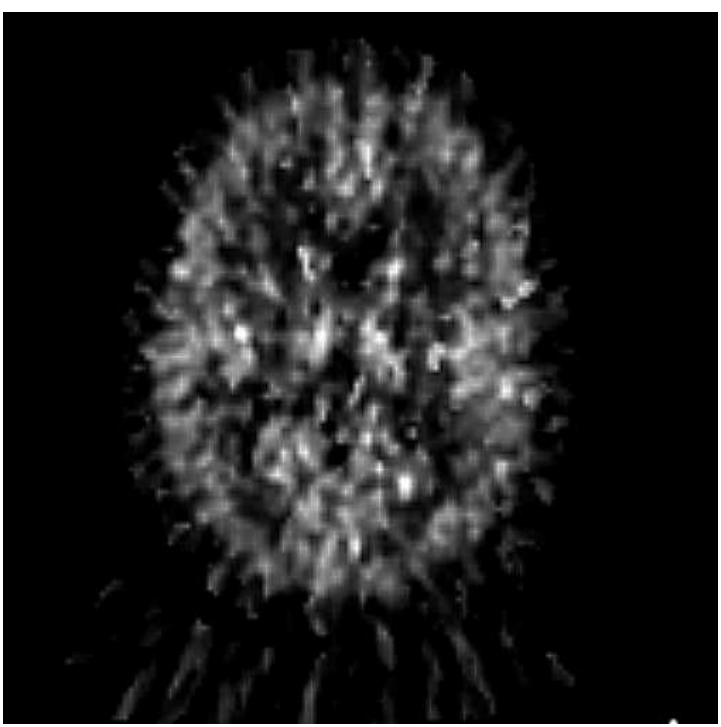

(b)

L1 RELATIVE ERROR IN GAUSSIAN APPROXIMATION

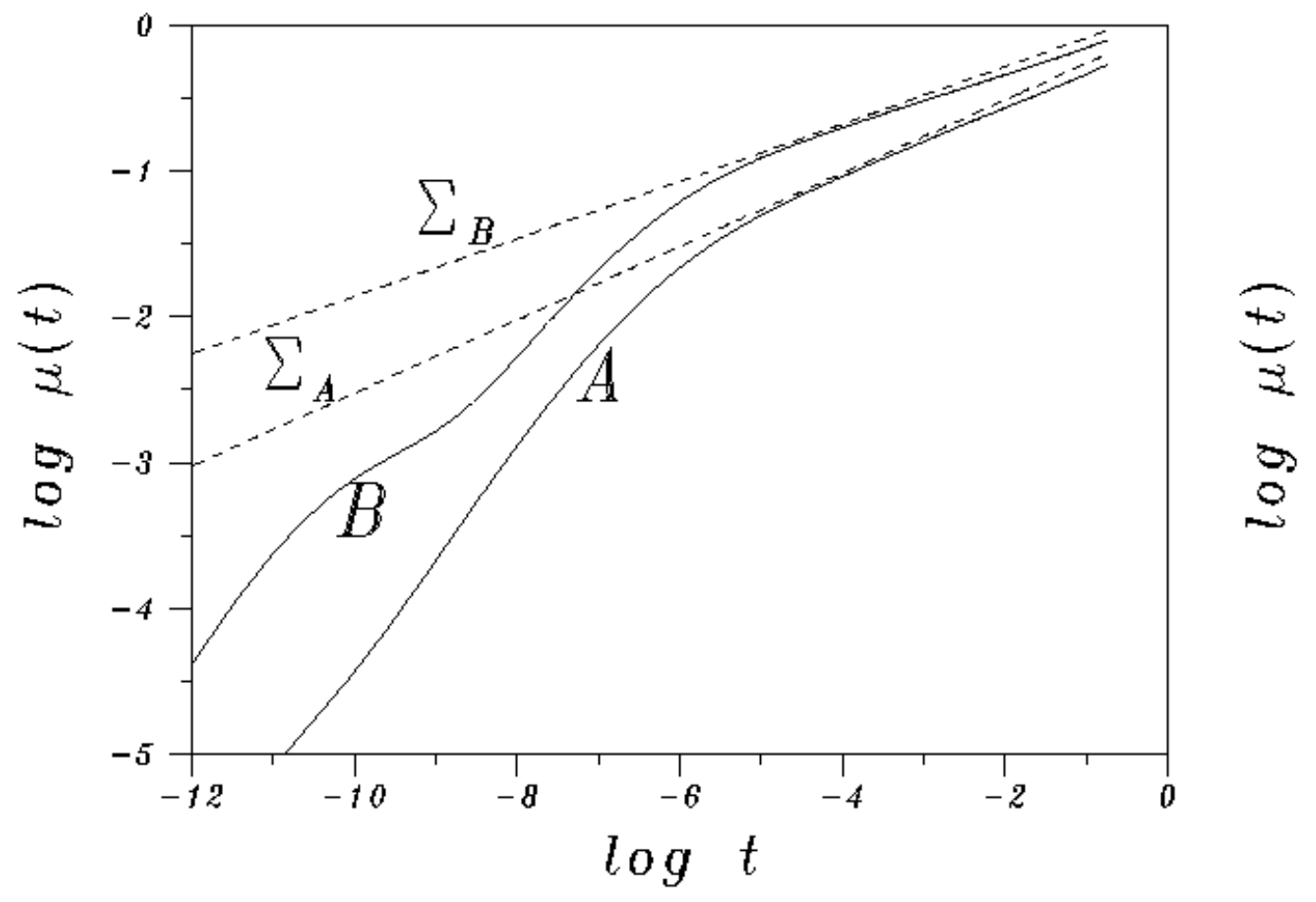

Figure 8: Evaluating the effectiveness......transverse functional PET... 


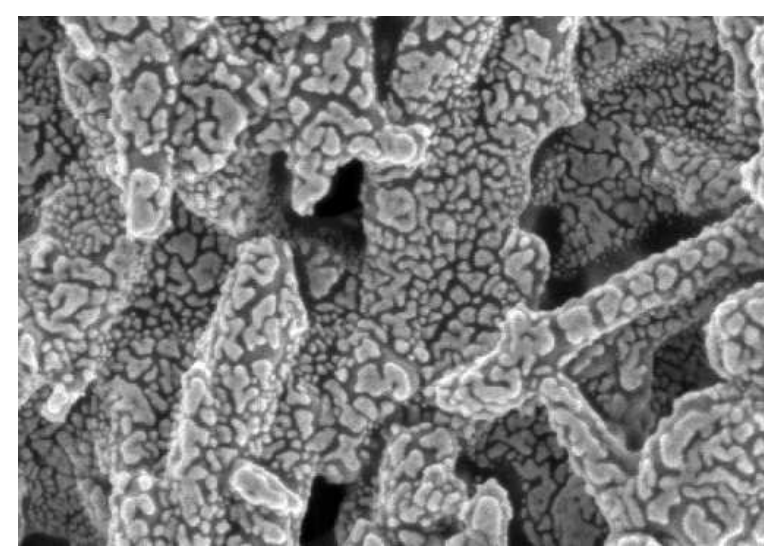

(a)

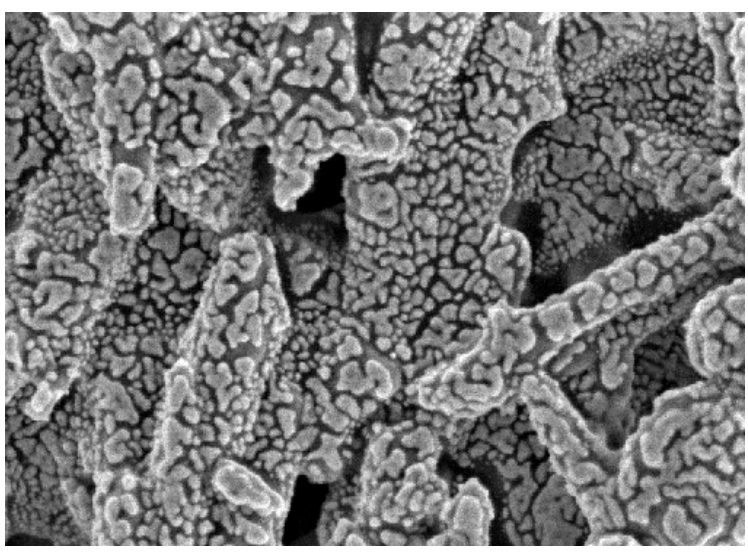

(b)

\section{L1 RELATIVE ERROR IN GAUSSIAN APPROXIMATION}

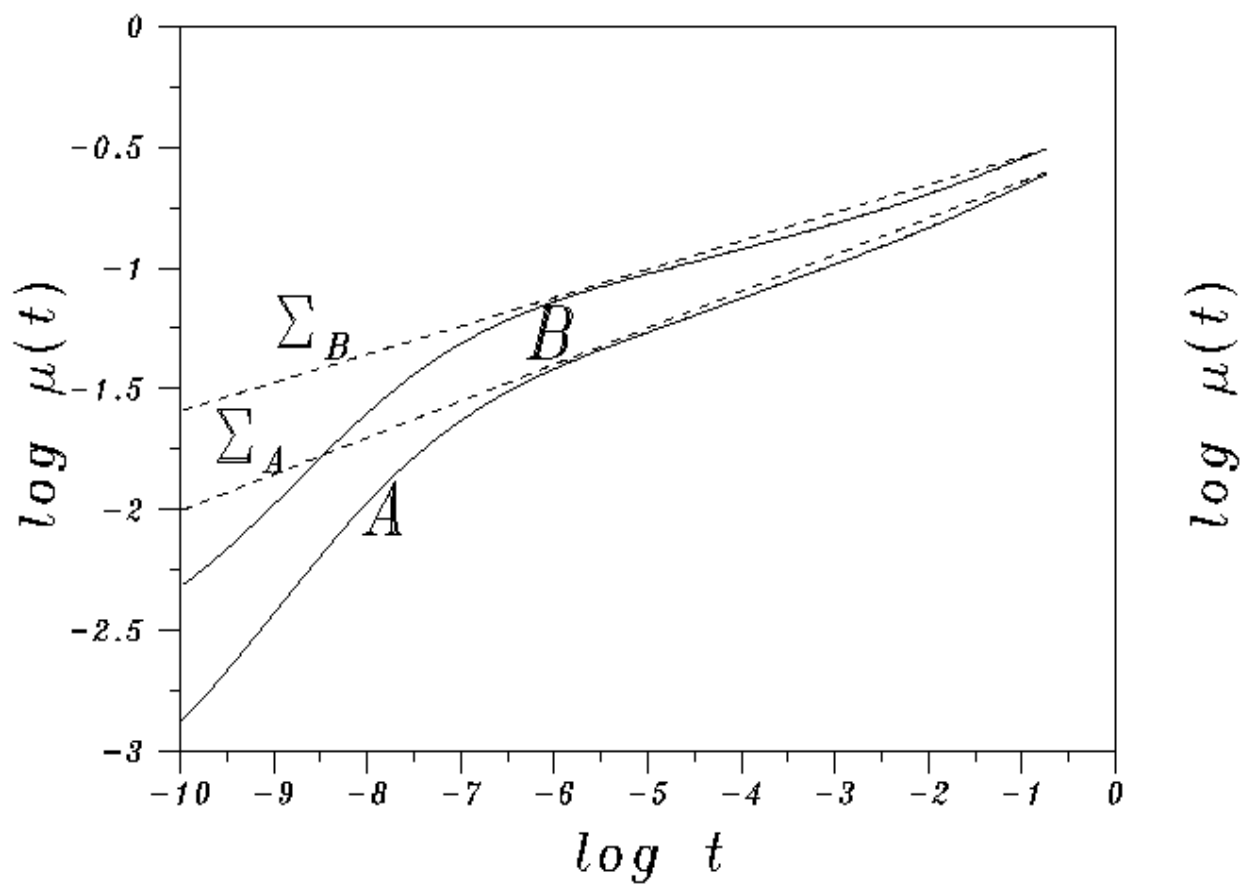

Figure 9: Evaluating the effectiveness.....magnetic tape sample.... 